\title{
De-projection of radio observations of axi-symmetric expanding circumstellar envelopes
}

\author{
P.T. Nhung ${ }^{1 \star}$, D.T. Hoai ${ }^{1}$, P. Tuan-Anh ${ }^{1}$, P. Darriulat ${ }^{1}$, T. Le Bertre $^{2}$, \\ J.M. Winters ${ }^{3}$, P.N. Diep ${ }^{1}$ and N.T. Phuong ${ }^{1}$ \\ ${ }^{1}$ Department of Astrophysics, Vietnam National Space Center (VNSC), Vietnam Academy of Science and Technology (VAST), \\ 18 Hoang Quoc Viet, Ha Noi, Viet Nam \\ ${ }^{2}$ LERMA, UMR 8112, CNRS and Observatoire de Paris, PSL Research University, 61 av. de l'Observatoire, F-75014 Paris, France \\ ${ }^{3}$ IRAM, 300 rue de la Piscine, Domaine Universitaire, F-38406 St. Martin d'Héres, France
}

Accepted XXX. Received YYY; in original form ZZZ

\begin{abstract}
The problem of de-projection of radio line observations of axi-symmetric expanding circumstellar envelopes is studied with the aim of easing their analysis in terms of physics models. The arguments developed rest on the remark that, in principle, when the wind velocity distribution is known, the effective emissivity can be calculated at any point in space. The paper provides a detailed study of how much this is true in practice. The wind velocity distribution assumed to be axi-symmetric and in expansion, is described by four parameters: the angles defining the orientation of the symmetry axis, an overall velocity scale and a parameter measuring the elongation (prolateness) of the distribution. Tools are developed that allow for measuring, or at least constraining, each of the four parameters. The use of effective emissivity as relevant quantity, rather than temperature and density being considered separately, implies important assumptions and simplifications meaning that the approach being considered here is only a preliminary to, and by no means a replacement for, a physics analysis accounting for radiative transfer and hydrodynamics arguments. While most considerations are developed using simulated observations as examples, two case studies (EP Aqr, observed with ALMA, and RS Cnc, with NOEMA) are presented that illustrate their usefulness in practical cases.
\end{abstract}

Key words: stars: circumstellar matter, AGB and post-AGB; methods: data analysis

\section{INTRODUCTION}

Recent years have seen high quality observations of molecular line emissions from evolved stars become available. Such observations, in particular from NOEMA (NOrthern Extended Millimeter Array) and from the Atacama Large Millimeter/submillimeter Array (ALMA), offer a spatial and spectral resolution calling for analysis methods making the best possible use of it. The present work addresses the case of expanding circumstellar envelopes of evolved stars, particularly stars populating the Asymptotic Giant Branch (AGB, for a recent review see Höfner \& Olofsson 2018, and references therein). Such envelopes have shapes that often evolve from spherical to axi-symmetric morphology, providing the seed for possibly more irregular configurations later observed in post-AGB stars and Planetary Nebulae. The physics governing the breaking of spherical symmetry is currently the subject of active research. In the case of binaries, attraction from the companion has been shown to play an important role. Yet, many unanswered questions, such as the role played by magnetic fields, remain to be elucidated.
The analysis of radio observations of molecular line emissions requires, as a preliminary, a de-projection in space. This is a largely under-determined problem: only two out of three position coordinates are measured, those in the sky plane; the position along the line of sight is unknown. And only one out of three velocity components is measured, that along the line of sight, from the observed Doppler shift. In a recent paper (Diep et al. 2016) we made general considerations on the problem of de-projection, with particular emphasis on the differentiation between expansion and rotation and on the use of Position-Velocity (PV) diagrams. We were then addressing issues related to both the physics of proto-stars and of evolved stars. In the present work, we concentrate instead on expanding circumstellar envelopes. The aim is to shed new light on the problem of de-projection in as simple terms as possible and to understand in depth the difficulties that its solution needs to face. To do so, we deliberately ignore complications such as arising from optical thickness or from the possible presence of rotation, and more generally from any form of departure from exact axi-symmetry and exact radial expansion. We mostly exploit the simplification offered by 
the constraint of axi-symmetry (one relation) and from the hypothesis of radial expansion (two relations), helping with the solution of the problem of de-projection.

The considerations that follow have no ambition at replacing the physics analysis required by an in-depth understanding of the physics mechanisms at play. They are simply meant as a useful preliminary step, providing helpful tools and possibly inspiring considerations on the issue of de-projection.

\section{THE FRAMEWORK}

We use coordinates $(x, y, z)$ attached to the sky plane and $\left(x^{\prime}, y^{\prime}, z^{\prime}\right)$ attached to the star (Figure 1). The $z$ axis is parallel to the line of sight, pointing away from Earth, while $x$ is pointing east and $y$ north. The $z^{\prime}$ axis is the symmetry axis of the star morphology and kinematics, making an angle $\varphi$ with the line of sight. Its projection on the sky plane makes an angle $\theta$ (position angle) with the $y$ axis, where $\theta$ is the angle between the $x$ axis and the $x^{\prime}$ axis, the latter taken to be in the $(x, y)$ plane. The transformation relations between the two systems of coordinates read

$$
\begin{aligned}
x^{\prime} & =x \cos \theta+y \sin \theta \\
y^{\prime} & =(-x \sin \theta+y \cos \theta) \cos \varphi+z \sin \varphi \\
z^{\prime} & =-(-x \sin \theta+y \cos \theta) \sin \varphi+z \cos \varphi \\
x & =x^{\prime} \cos \theta-\left(y^{\prime} \cos \varphi-z^{\prime} \sin \varphi\right) \sin \theta \\
y & =\left(y^{\prime} \cos \varphi-z^{\prime} \sin \varphi\right) \cos \theta+x^{\prime} \sin \theta \\
z & =y^{\prime} \sin \varphi+z^{\prime} \cos \varphi
\end{aligned}
$$

However, in much of what follows, we redefine the $y$ axis as the projection of the star axis on the sky plane, which is equivalent to setting $\theta=0$. In this case $x=x^{\prime}$ and the transformation relations between $(y, z)$ and $\left(y^{\prime}, z^{\prime}\right)$ read:

$$
\begin{array}{cl}
y^{\prime}=y \cos \varphi+z \sin \varphi & y=y^{\prime} \cos \varphi-z^{\prime} \sin \varphi \\
z^{\prime}=-y \sin \varphi+z \cos \varphi & z=y^{\prime} \sin \varphi+z^{\prime} \cos \varphi
\end{array}
$$

In order to illustrate our arguments as simply as possible we use a model (in the remainder of the article we refer to it as "the simple model") in which the wind velocity is radial and independent of the distance from the star with a dependence on star latitude $\alpha$ of the form

$V=V_{\text {pole }} \sin ^{2} \alpha+V_{e q} \cos ^{2} \alpha=V_{0}(1-\lambda \cos 2 \alpha)$

with the polar velocity $V_{\text {pole }}=V_{0}(1+\lambda)$ and the equatorial velocity $V_{e q}=V_{0}(1-\lambda)$ (Figure 2 left). For $\lambda$ between 0 and 1 one obtains prolate velocity distributions (bipolar outflow) and for $\lambda$ between -1 and 0 oblate velocity distributions (equatorial outflow), $\lambda=0$ corresponding to an isotropic (spherical) velocity distribution. Here $V_{0}$ defines the velocity scale but its precise value is irrelevant; in practice, we set it at $5 \mathrm{~km} \mathrm{~s}^{-1}$ for the purpose of illustration. The Doppler velocity at space point $(x, y, z)$ is

$V_{z}=V \sin \zeta$

where $\zeta$ is the angle between the $(x, y, z)$ direction and the plane of the sky. We also define $R=\sqrt{x^{2}+y^{2}}=z / \tan \zeta$ and $r=\sqrt{R^{2}+z^{2}}=z / \sin \zeta=R / \cos \zeta$.

From Relations 2, 3 and 4, using $\sin \alpha=z^{\prime} / r$ and replacing

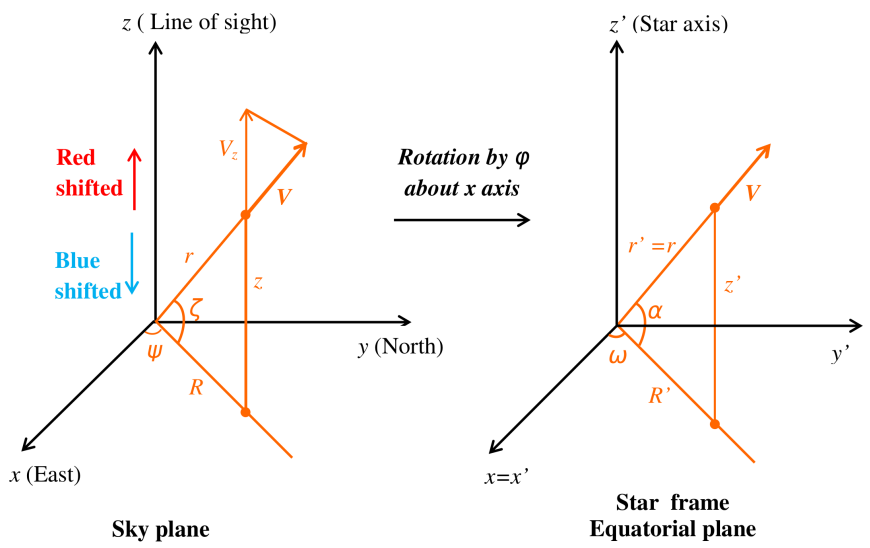

Figure 1. Coordinate systems for $\theta=0$. Relations 5 transform from the sky frame (left) to the star frame (right).

$z^{\prime}$ by its expression in Relation 2 we can express the Doppler velocity as a function of $\zeta, \varphi$ and $\psi$, defined as the position angle measured with respect to the projection of the star axis on the sky plane, $y=R \cos \psi$ :

$$
\begin{aligned}
V_{z}= & V_{0}(z / r)\left[1-\lambda\left(1-2 \sin ^{2} \alpha\right)\right] \\
= & V_{0}(z / r)\left[1-\lambda+2 \lambda(z \cos \varphi-y \sin \varphi)^{2} / r^{2}\right] \\
V_{z}= & V_{0} \sin \zeta\left[1-\lambda+2 \lambda(\sin \zeta \cos \varphi-\cos \zeta \cos \psi \sin \varphi)^{2}\right] \\
= & V_{0}\left[(1-\lambda) \sin \zeta+2 \lambda \sin ^{3} \zeta \cos ^{2} \varphi\right. \\
& -4 \lambda \sin ^{2} \zeta \cos \zeta \sin \varphi \cos \varphi \cos \psi \\
& \left.+2 \lambda \sin \zeta \cos ^{2} \zeta \sin ^{2} \varphi \cos ^{2} \psi\right]
\end{aligned}
$$

Taking the derivative along the line of sight $(R$ and $\psi$ being fixed) and using the identities $d / d z=[d / d(\sin \zeta)][d(\sin \zeta) / d z]=r^{-1}\left(\cos ^{2} \zeta\right) d / d(\sin \zeta)$ and $d(\cos \zeta) / d(\sin \zeta)=-\tan \zeta$ we obtain:

$r d V_{z} / d z=V_{0}\left[(1-\lambda) \cos ^{2} \zeta+2 \lambda P\right]$

with

$$
\begin{aligned}
P= & 3 \cos ^{2} \varphi \sin ^{2} \zeta \cos ^{2} \zeta \\
& -\cos \psi \sin 2 \varphi \cos \zeta \sin \zeta\left(2 \cos ^{2} \zeta-\sin ^{2} \zeta\right) \\
& +\cos ^{2} \psi \sin ^{2} \varphi \cos ^{2} \zeta\left(\cos ^{2} \zeta-2 \sin ^{2} \zeta\right)
\end{aligned}
$$

Radio astronomy measurements are in the form of a data-cube with elements $f\left(x, y, V_{z}\right)$ measuring the brightness along the line $(x, y)$ normal to the sky plane at Doppler velocity $V_{z}$. It is convenient to define the effective emissivity at space point $(x, y, z)$ as

$\rho(x, y, z)=f\left(x, y, V_{z}\right) d V_{z} / d z$

and the measured intensity as

$F(x, y)=\int f\left(x, y, V_{z}\right) d V_{z}=\int \rho(x, y, z) d z$

As mentioned in the introduction, the assumption of pure radial velocity and of axi-symmetry should help with the de-projection of the effective emissivity using Relation 6. How much this is true in practice is the subject of the present article. 

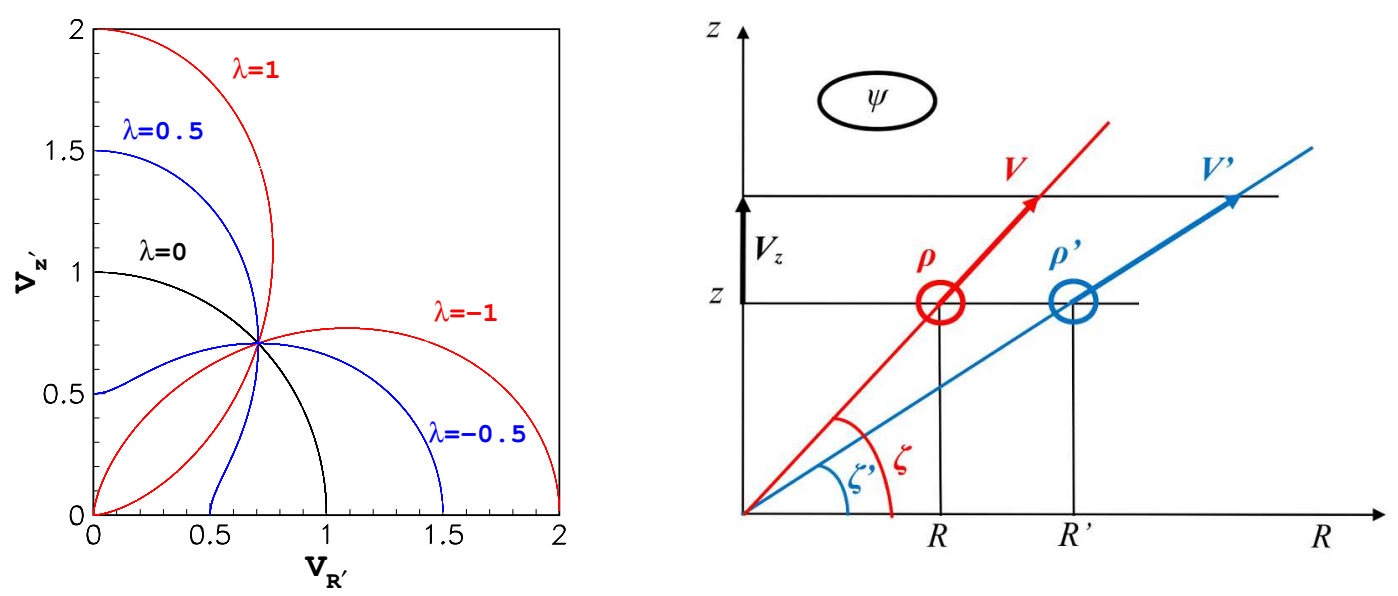

Figure 2. Left: polar diagrams of $V$ in the upper meridian quadrant ( $R^{\prime}$ in abscissa and $z^{\prime}$ in ordinate) for different values of the prolateness parameter $\lambda$. Right: Deprojection in the $(R, z)$ plane (see text).
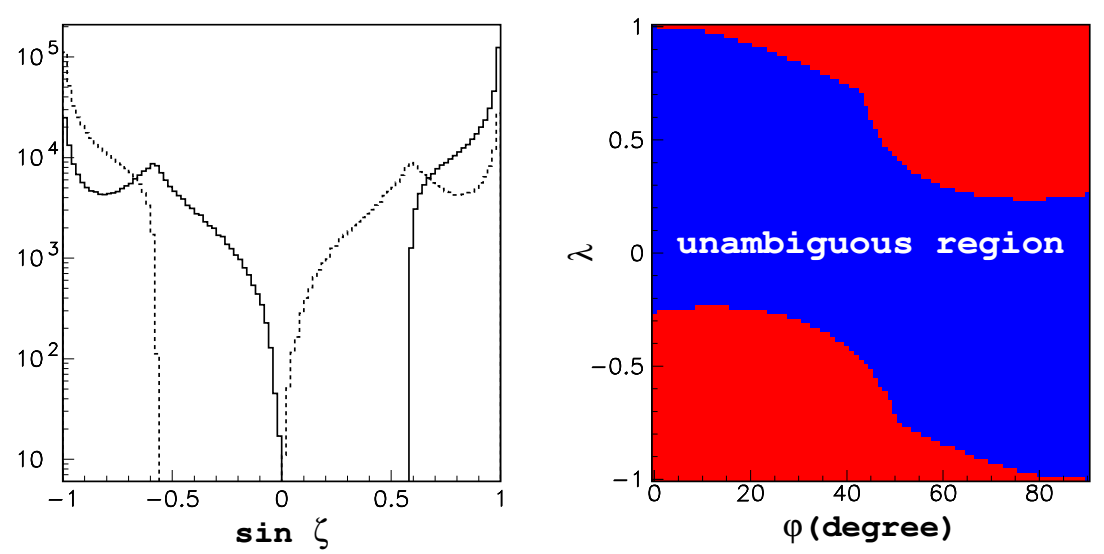

Figure 3. Left: $\sin \zeta$ (abscissa) distribution of the extrema of the $V_{z}$ for a million of uniformly distributed $(\lambda, \varphi, \psi)$ triplets. Dashed line is for $\lambda<0$ (oblate) and solid line for $\lambda>0$ (prolate). Right: region (in blue) of the $\lambda$ (ordinate) vs $\varphi$ (abscissa) plane where de-projection is unambiguous, $z$ increasing monotonically with $V_{z}$ in the region $|\sin \zeta|<0.9$.

A number of symmetries are apparent in Relations 5. Changing $\varphi$ in $-\varphi$ and $\psi$ in $180^{\circ}-\psi$ leaves $V_{z}$ invariant, so does also changing $\psi$ in $-\psi$; changing $\varphi$ in $180^{\circ}-\varphi$ and $\zeta$ in $-\zeta$ changes $V_{z}$ in $-V_{z}$. Accordingly, it is sufficient to limit the ranges of $\varphi$ and $\psi$ to respectively $\left[0^{\circ}, 90^{\circ}\right]$ and $\left[0^{\circ}, 180^{\circ}\right]$.

A remarkable consequence of the above relations is the ability to estimate the $r$-dependence of the effective emissivity independently from the form chosen for the wind velocity $\left(V_{0}, \lambda, \varphi\right)$ as long as $V$ does not depend on $r$ (Figure 2 right). Indeed, consider a line passing by the star and having direction $(\psi, \zeta)$, namely making an angle $\zeta$ with the plane of the sky and projecting on it at position angle $\psi$; this line corresponds to a single value of $V_{z}$, independent of both $R$ and $r$ as long as the radial expansion velocity is constant on it, namely independent of $r$. Therefore, as long as both the real wind velocity, $V(\psi, \zeta)$, and the wind velocity used for de-projection, $V^{\prime}(\psi, \zeta)$ are both independent of $r$ (but depend on $\psi$ and $\zeta$, generally in different ways) the de-projected line is also a straight line, having the same projection on the plane of the sky; its direction $\left(\psi^{\prime}, \zeta^{\prime}\right)$ is related to the direction $(\psi, \zeta)$ by the relations $\psi^{\prime}=\psi$ and $V^{\prime}\left(\psi, \zeta^{\prime}\right) \sin \zeta^{\prime}=V(\psi, \zeta) \sin \zeta$. De-projection simply transforms $\zeta$ into $\zeta^{\prime}$ and the ratio $z^{\prime} / z$ is independent of $R$. As a result, the true effective emissivity $\rho$ and the de-projected effective emissivity $\rho^{\prime}$ have the same dependence on $R$ on each of these two lines, $(\psi, \zeta)$ for the former and $\left(\psi, \zeta^{\prime}\right)$ for the latter: they are proportional to a same data-cube element $f\left(x, y, V_{z}\right)$, independently from $R$. To the extent that the true wind velocity and the wind velocity used for deprojection are not too different, the $r$-dependence of the deprojected effective emissivity is similar to the $r$-dependence of the true effective emissivity, both being dominated by the $R$ dependence of the Doppler velocity spectrum. 

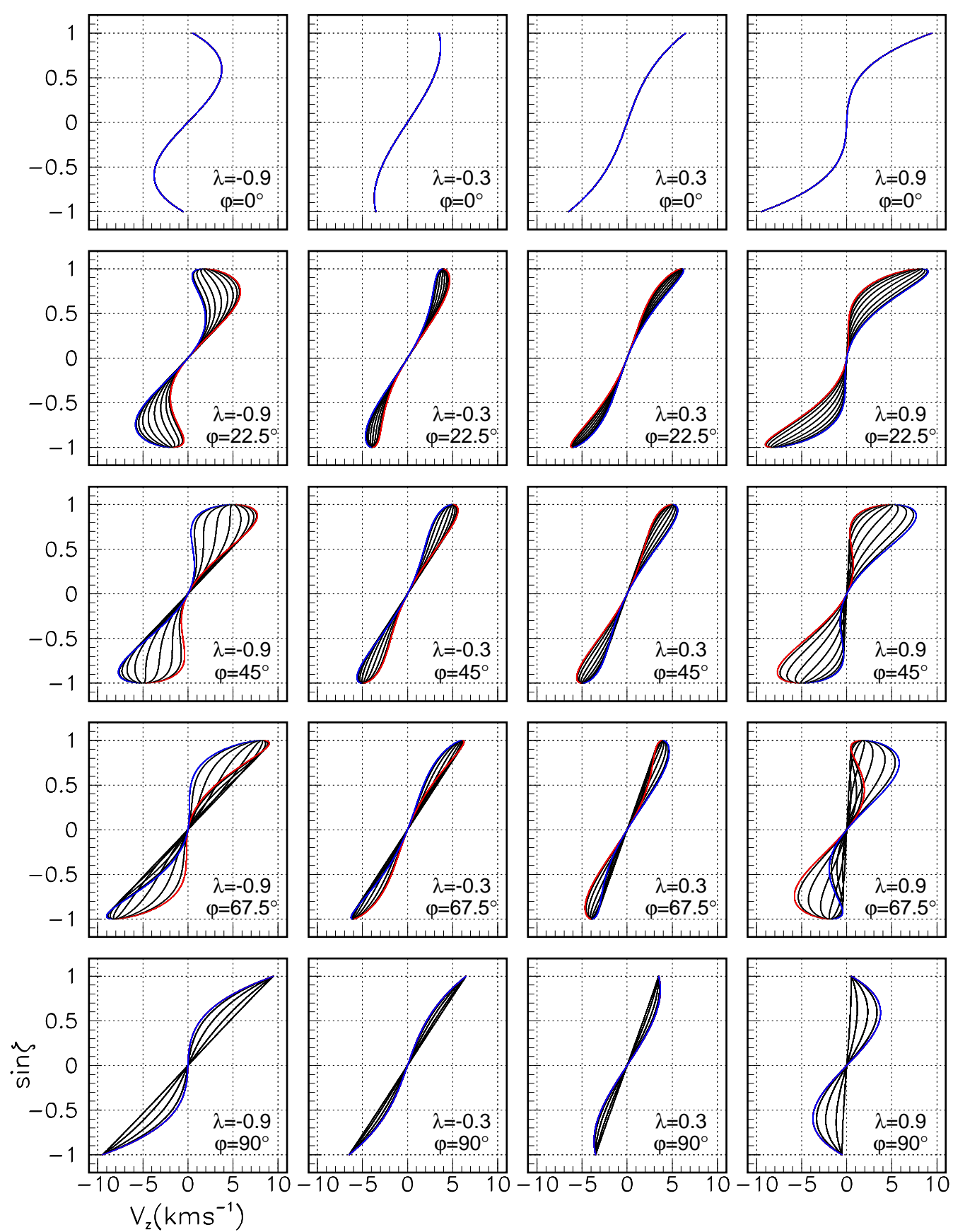

$\mathrm{V}_{z}\left(\mathrm{kms}^{-1}\right)$

Figure 4. Dependence of $\sin \zeta$ (ordinate) on $V_{z}$ (abscissa) for different values of $\psi$ (from $0^{\circ}$ to $180^{\circ}$ in steps of $20^{\circ}$ ). Panels are in five rows of $\varphi$ (up down from $0^{\circ}$ to $90^{\circ}$ in steps of $22.5^{\circ}$ ) and in columns of $\lambda$ (left to right from -0.9 to +0.9 in steps of 0.6 ).

\section{GENERAL CONSIDERATIONS}

De-projection implies using Relation 6 to calculate the effective emissivity $\rho(x, y, z)$ from the measured brightness $f\left(x, y, V_{z}\right)$ by associating to each value $V_{z}$ of the measured Doppler velocity spectrum a point $(x, y, z)$ in space. This is only possible if the relation giving $V_{z}$ as a function of $z$ can be inverted into a relation giving $z$ as a function of $V_{z}$. In general, an extremum of the dependence of $V_{z}$ on $z$ will generate in its vicinity two values of $z$ for a same value of $V_{z}$. In principle, this should prevent de-projection as one does not know how to share the brightness measured at Doppler velocity $V_{z}$ between the two corresponding space points; this issue will be discussed in some detail in Section 6 . As the relation between $z$ and $\sin \zeta$ is one-to-one, $z=R \tan \zeta=R \sin \zeta / \sqrt{1-\sin ^{2} \zeta}$, the extrema of $V_{z}$ vs $z$ are the same as of $V_{z}$ vs $\sin \zeta$.

In order to obtain some insight into this question, 

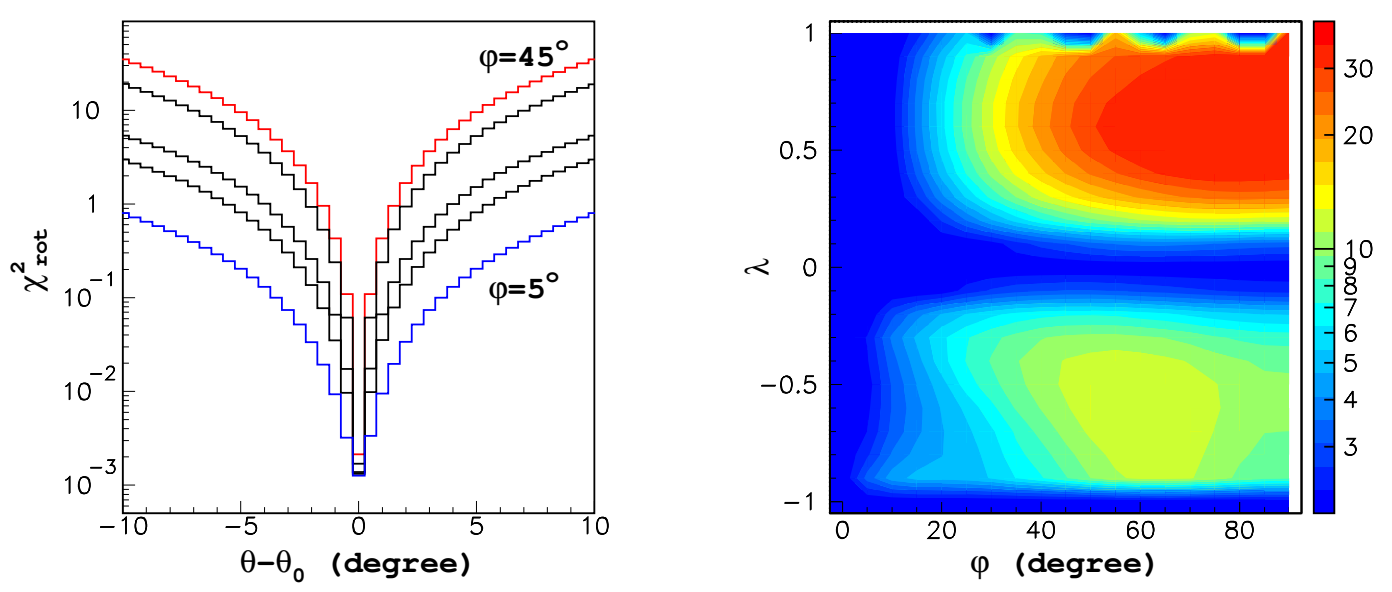

Figure 5. Left: dependence of $\chi_{\text {rot }}^{2}$ (ordinate) on $\theta-\theta_{0}$ (degrees, abscissa) for $\lambda=0.5$ and $\varphi=5^{\circ}, 10^{\circ}, 15^{\circ}, 30^{\circ}, 45^{\circ}$ (from down up). Right: dependence of $S_{\text {rot }}$ on $\lambda$ (ordinate) and $\varphi$ (abscissa).

we consider a sample of uniformly distributed $(\lambda, \varphi, \psi)$ triplets. Figure 3 (left) displays the values of $\sin \zeta$ at which an extremum of $V_{z}$ vs $z$ is found; they concentrate near $\sin \zeta= \pm 1$. To see it more directly, we display in Figure 4 a set of representative functions $\sin \zeta$ vs $V_{z}$. For $\sin \zeta= \pm 1, V_{z}= \pm V_{0}(1+\lambda \cos 2 \varphi)$ and when $\sin \zeta$ departs from $\pm 1, V_{z}$ increases or decreases depending on the sign of $\lambda \sin 2 \varphi \cos \psi$. Globally, $V_{z}$ must increase with $z$; if it starts in the wrong direction, it needs to turn back and one obtains an extremum. When such extrema are in the vicinity of $\sin \zeta= \pm 1$, they are not too harmful for de-projection: they simply mean that each end region of the Doppler velocity spectrum has to be assigned a broad range of $\sin \zeta$ values in the vicinity of the line of sight. However, the farther away they are from $\sin \zeta= \pm 1$, the larger the fraction of the Doppler velocity spectrum that becomes unsuitable for de-projection. In a majority of cases $(68 \%)$ there is no "harmful" extremum, defined as having $|\sin \zeta|>0.9$.

The right panel of Figure 3 displays the region of the $(\lambda, \varphi)$ plane where no "harmful" extremum occurs, namely where $d V_{z} / d z$ does not cancel in the $|\sin \zeta|<0.9$ region. In this region, as clearly illustrated in Figure 4, large values of $\left|V_{z}\right|$ are associated with large values of $|\sin \zeta|$ and deprojection can be unambiguously performed as long as $V_{0}$ is large enough. In the complementary ambiguous region, on the contrary, the larger values of $\left|V_{z}\right|$ are associated with intermediate values of $|\sin \zeta|$, the larger values of $|\sin \zeta|$ being now associated with lower values of $\left|V_{z}\right|$ : the Doppler velocity spectrum is folded on itself, the larger values of $\left|V_{z}\right|$ being associated with two values of $|\sin \zeta|$, making de-projection ambiguous and unreliable. The strong qualitative difference between the two regions will be seen to play a major role in the arguments developed in the present study.

\section{POSITION ANGLE OF THE PROJECTION OF THE STAR AXIS ON THE SKY PLANE}

The invariance of Relations 5 when $\psi$ changes sign implies an exact symmetry of the data-cube with respect to the plane of position angle $\theta$, perpendicular to the sky plane and containing the star axis and the line of sight. It is indeed a consequence of axi-symmetry and is expected to apply for any axi-symmetric model, not just the simple model used here for illustration. In principle, the symmetry plane, and therefore the value of $\theta$, can be simply found by minimizing the quantity

$\chi_{\text {rot }}^{2}=\sum\left[\left(f\left(x, y, V_{z}\right)-f\left(x^{*}, y^{*}, V_{z}\right)\right]^{2} /(\Delta f)^{2}\right.$

where $\left(x^{*}, y^{*}\right)$ is the symmetric of $(x, y)$ with respect to direction $\theta$ :

$x^{*}=-(x \cos 2 \theta+y \sin 2 \theta) \quad y^{*}=-(x \sin 2 \theta-y \cos 2 \theta)$

and where $\Delta f$ is the uncertainty on the $f$ measurement. In the present section we use a simulation having a wind of the form given in Relation 3 and we simply take as $\Delta f$ the quadratic sum of the rms deviations of $f$ from its mean in the vicinity of each of $\left(x, y, V_{z}\right)$ and $\left(x^{*}, y^{*}, V_{z}\right)$.

As an illustration of the procedure, Figure 5 (left) shows the dependence of $\chi_{\text {rot }}^{2}$ on $\theta-\theta_{0}$ for $\lambda=0.5$ and various values of $\varphi$, with $\theta_{0}$ being the value of $\theta$ used to produce the simulated effective emissivity. The angle $\theta$ is undefined in two obvious cases: for an isotropic velocity distribution, $\lambda=0$, and for a star axis parallel to the line of sight, $\varphi=0$; the latter case is a trivial effect of geometry, the elementary solid angle being $d \Omega=\sin \varphi d \theta d \varphi$ rather than simply $d \theta d \varphi$. Indeed, the dependence of $\chi_{\text {rot }}^{2}$ on $\theta-\theta_{0}$ is observed to display a steep minimum at 0 as long as $\lambda$ and $\varphi$ are not too close from zero.

In order to assess the accuracy of the $\theta$ measurement, we calculate the relative increase $S_{\text {rot }}$ of $\chi_{\text {rot }}^{2}$ associated with a shift of $\pm 1^{\circ}$ from $\theta=\theta_{0}: S_{\text {rot }}=1 / 2\left[\chi_{\text {rot }}^{2}(\theta=\right.$ 


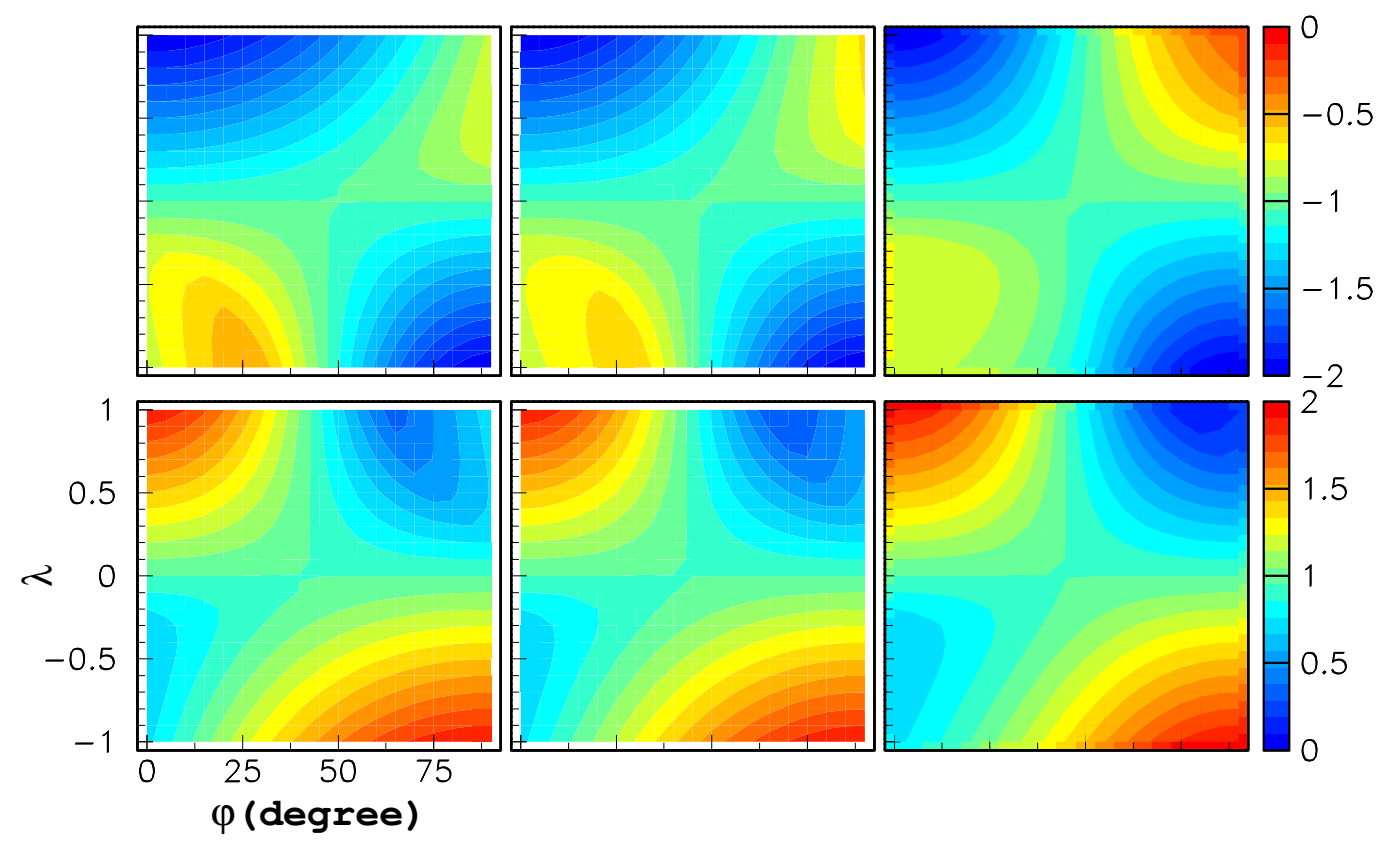

Figure 6. Maps of $V_{z \min } / V_{0}$ (upper panels) and $V_{z \max } / V_{0}$ (lower panels) in the $\lambda$ (ordinate) vs $\varphi$ (abscissa) plane for $0<\psi<30^{\circ}$ (left), $30^{\circ}<\psi<60^{\circ}$ (middle) and $60^{\circ}<\psi<90^{\circ}$ (right).

$\left.\left.\theta_{0}-1^{\circ}\right)+\chi_{\text {rot }}^{2}\left(\theta=\theta_{0}+1^{\circ}\right)\right] / \chi_{\text {rot }}^{2}\left(\theta=\theta_{0}\right)$. The larger $S_{\text {rot }}$ and the farther away from unity, the better defined is the value of $\theta$. Figure 5 (right) displays the dependence of $S_{\text {rot }}$ on $\lambda$ and $\varphi$. Its actual value when applied to real observations depends on the relevant uncertainties, usually caused by the lumpiness of the effective emissivity rather than by noise; but its behaviour in the $(\lambda, \varphi)$ plane remains essentially the same as found here. However, a systematic rather than random deviation from axi-symmetry may affect the measurement of $\theta$, as was already discussed in the case of rotation by Diep et al. (2016). However, a systematic violation of axi-symmetry by the effective emissivity or by the wind radial expansion velocity of the form $1+\epsilon \cos \left(\omega-\omega_{0}\right)$ does not shift the value of $\theta$ but simply broadens the minimum of $\chi_{\text {rot }}^{2}$, because, contrary to rotation, it distorts in a same way the red-shifted and blue-shifted hemispheres.

\section{MAGNITUDE OF THE WIND VELOCITIES: WIDTH AND OFFSET OF THE DOPPLER VELOCITY SPECTRUM}

In general, the radial wind velocity $V$ is confined between two finite values; in the case of the simple model, these are $V_{e q}$ and $V_{\text {pole }}$, respectively $V_{0}(1-\lambda)$ and $V_{0}(1+\lambda)$. However, at a given point $(x, y)$ in the sky plane, the measured Doppler velocity varies between two values $V_{z \min }$ and $V_{z \max }$ that are not simply related to the above. Yet, when choosing a velocity distribution with which to de-project the effective emissivity, it is essential to have some idea of its scale, meaning the value of $V_{0}$ in the case of the simple model. In principle, having chosen a pair $(\lambda, \varphi)$ for de-projection, the values of $V_{z \min } / V_{0}$ and $V_{z \max } / V_{0}$ that they generate in each pixel are known. One should then choose for $V_{0}$ the ratio between the measured values of $V_{z \min }$ and $V_{z \max }$ and the model values of $V_{z \min } / V_{0}$ and $V_{z \max } / V_{0}$. The values of $V_{z \min } / V_{0}$ and $V_{z \max } / V_{0}$ depend on the pixel. In general, different pixels produce different values of $V_{0}$ and their mean, or better their maximum, should be retained for de-projection. Note that changing $z$ in $-z$ and $\cos \psi$ in $-\cos \psi$ changes $V_{z}$ in $-V_{z}$ but leaves $d V_{z} / d z$ invariant; therefore it changes $V_{z \min }$ in $-V_{z \max }$ and $V_{z \max }$ in $-V_{z \min }$ : it is sufficient to confine $\psi$ to the $\left[0^{\circ}, 90^{\circ}\right]$ interval. Figure 6 displays the maps of $V_{z m i n} / V_{0}$ and $V_{z \max } / V_{0}$ in the $(\lambda, \varphi)$ plane for three intervals of $\psi$, each $30^{\circ}$ wide, covering between $0^{\circ}$ and $90^{\circ}$. Qualitatively, the dependence on $\psi$ is weak. The main features reflect the effect of the width of the Doppler velocity spectrum, which is large on the descending and small on the ascending diagonal. On the former, from $(\lambda, \varphi)=\left(+1,0^{\circ}\right)$ to $\left(-1,90^{\circ}\right)$, namely from a bipolar outflow parallel to the line of sight to an equatorial outflow having its axis in the sky plane, $V_{z \min } / V_{0}$ varies between -1 and -2 and $V_{z \max } / V_{0}$ between 1 and 2. On the latter, from $(\lambda, \varphi)=\left(-1,0^{\circ}\right)$ to $\left(+1,90^{\circ}\right)$, namely from a bipolar to an equatorial outflow, both in the sky plane, $V_{z \min } / V_{0}$ varies between -1 and 0 and $V_{z \max } / V_{0}$ between 0 and 1 .

When de-projecting the effective emissivity using a $(\lambda, \varphi)$ pair of parameters, agreement between the values obtained in each pixel for $V_{0}$ as a function of $\psi$ and $R$ is a useful indicator of the suitability of the particular $(\lambda, \varphi)$ pair to describe the observations. The ratio $Q=V_{r m s} / V_{\text {mean }}$ between 

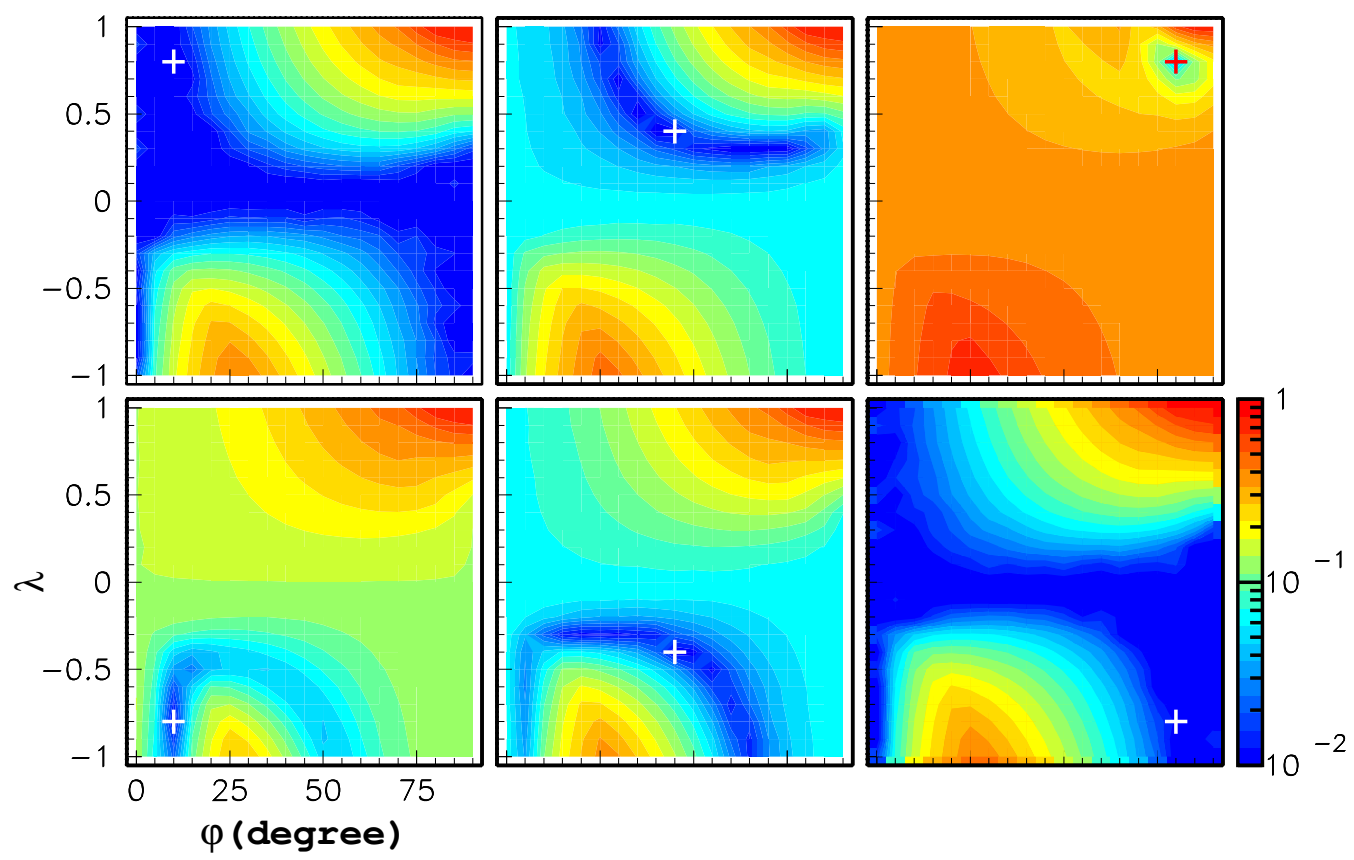

Figure 7. Dependence of $Q$ on the values of $\lambda$ (ordinate) and $\varphi$ (abscissa) used in de-projection for a few simulated wind configurations of the form $V\left(\mathrm{~km} \mathrm{~s}^{-1}\right)=5(1-\lambda \cos 2 \alpha)$ indicated as a cross. From left to right, the values of $(\lambda, \varphi)$ used in the simulation are $( \pm 0.8$, $\left.10^{\circ}\right),\left( \pm 0.4,45^{\circ}\right)$ and $\left( \pm 0.8,80^{\circ}\right)$ respectively.

their rms value $V_{r m s}$ and their mean value $V_{\text {mean }}$, calculated over the whole image, when too large, can be used to reject unsuitable wind configurations: the study of the dependence on position angle of the width and offset of the Doppler velocity spectrum is not only a tool to obtain an evaluation of the scale of the space velocity (here $V_{0}$ ) but also, in principle, to reject unsuitable values of the $(\lambda, \varphi)$ pair. This is illustrated in Figure 7, which displays the dependence of $Q$ on $(\lambda, \varphi)$ for a few typical simulated wind configurations. In all cases a steep minimum of $Q$ is obtained in a narrow region of the $(\lambda, \varphi)$ plane containing the values used in the simulation, suggesting that an important fraction of the $(\lambda, \varphi)$ plane could be eliminated by simply requiring $Q$ not to exceed some threshold. In practice, however, as will be seen in Section 7, the minimum of $Q$ is much less steep for real than for simulated data.

We remark that if $V_{0}$ is slightly overestimated, its largest values will de-project in a region of the observed Doppler velocity spectrum where there are no data and will accordingly set the de-projected effective emissivity to zero. On the contrary, if $V_{0}$ is slightly underestimated, the larger values of the observed Doppler velocity spectrum will be ignored. It is therefore important, in practice, to make sure that the obtained value of $V_{0}$ is optimal and, if necessary, to fine-tune it.

\section{MEASURING THE PROLATENESS PARAMETER $\lambda$ AND THE INCLINATION $\varphi$ OF THE STAR AXIS WITH RESPECT TO THE LINE OF SIGHT}

The results obtained in the preceding two sections are largely independent from the particular form of the dependence of the effective emissivity on stellar latitude. They provide reliable estimates of the scale $V_{0}$ of the space velocity distribution and of the position angle $\theta$ of the projection of the star axis on the sky plane. Moreover, they eliminate regions of the $(\lambda, \varphi)$ plane that are unsuitable for de-projection. We are then left with two parameters, $\lambda$ and $\varphi$, to be measured in the case of the simple model. In the general case, several parameters will be necessary to describe the wind velocity in place of the pair $\left(V_{0}, \lambda\right)$ and $\lambda$ must be seen as a measure of the effective prolateness of the wind velocity distribution.

In the present section, we exploit the constraint resulting from the requirement of axi-symmetry of the effective emissivity to help with the measurement of the $(\lambda, \varphi)$ pair. This constraint is less useful in some cases than in others. For example, in the case of a wind velocity having its axis parallel to the line of sight any pair $(\lambda, \varphi=0)$ used in deprojection will produce an axi-symmetric effective emissivity, independently from the value assumed for $\lambda$.

In order to understand under which conditions the constraint of axi-symmetry is strong, we use a simple measure of the amount of axi-symmetry of the de-projected effective 

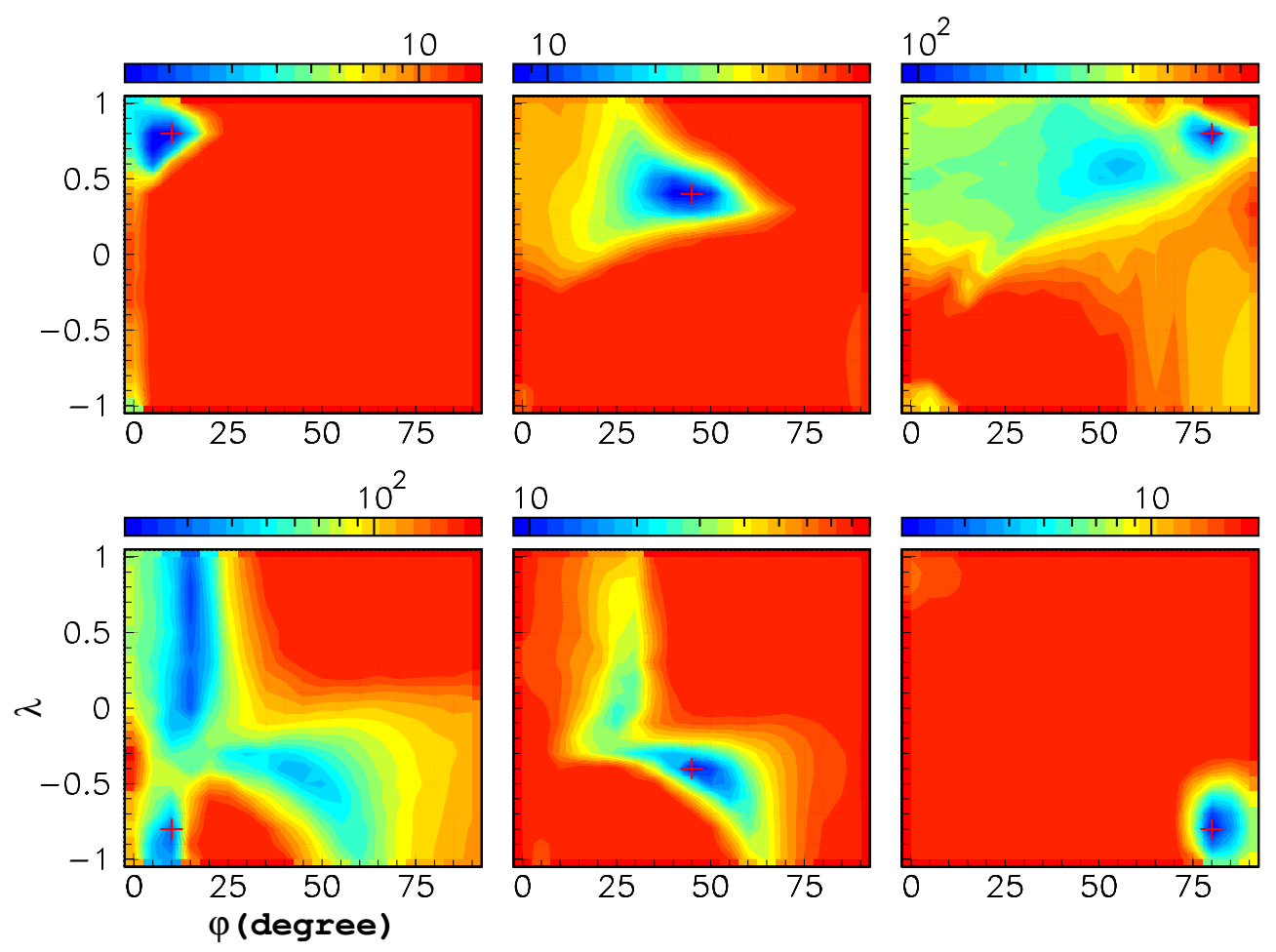

Figure 8. Dependence of $\chi_{a x i}^{2}$ on the values of $\lambda$ (ordinate) and $\varphi$ (abscissa) used in de-projection for the same simulated wind configurations indicated as a cross as in Figure 7. In each panel, the colour scale extends from minimum to ten times minimum.

emissivity about axis $(\theta, \varphi)$ :

$\chi_{a x i}^{2}=\sum[\rho(r, \alpha, \omega)-<\rho(r, \alpha)>]^{2} /(\Delta \rho)^{2}$

where $\omega$ is the stellar longitude $\left(x^{\prime}=r \cos \alpha \cos \omega, y^{\prime}=\right.$ $r \cos \alpha \sin \omega$ and $\left.\tan \omega=y^{\prime} / x^{\prime}\right) ; \rho(r, \alpha, \omega)$ is the effective emissivity de-projected using a wind configuration of axis $(\theta, \varphi)$, effective prolateness $\lambda$ and velocity scale $V_{0}$ estimated from the procedure described in the preceding section; $<\rho(r, \alpha)\rangle$ is its mean value at $(r, \alpha)$, averaged over longitude $\omega ; \Delta \rho$ is the uncertainty attached to the evaluation of $\rho$. The sum extends over the whole space over which measurements are available. The evaluation of $\Delta \rho=\Delta f\left|d V_{z} / d z\right|$, where $\Delta f$ is the uncertainty on the de-projected data-cube element, uses here the same estimate of $\Delta f$ as used for $\chi_{\text {rot }}^{2}$ in Section 4, namely the rms deviation of $f$ from its mean in the vicinity of the de-projected space point. In real cases, however, the definition of $\Delta f$ is delicate and needs to account for both experimental uncertainties and uncertainties attached to the de-projection; we discuss this point in more detail in Section 7. In practice the calculation of $\chi_{a x i}^{2}$ proceeds as follows: one chooses a circle having the star axis as axis and defined by its position $\left(R^{\prime}, z^{\prime}\right)$ in the star frame and scans over it by varying the stellar longitude $\omega$, each time calculating the space position $(x, y, z)$ and the associated value of $V_{z}$ for the values of $\left(V_{0}, \lambda, \varphi\right)$ of the axi-symmetric wind used in de-projection.

The main weakness of this method is its mishandling of cases where two different values of $z / r$ are associated with a same bin of Doppler velocity. Such bins contain contributions from each of the two regions but the de-projection algorithm wrongly assigns their total content to each of the two regions, generating double-counting. As was already remarked in Section 3, one does not know how to share it between the two regions and such bins are unsuitable for de-projection. We have seen in Section 3 that for $z / r= \pm 1, V_{z}= \pm V_{0}(1+\lambda \cos 2 \varphi)$ and that when $z / r$ departs from $\pm 1, V_{z}$ increases or decreases depending on the sign of $\lambda \sin 2 \varphi \cos \psi$. Rather than simply ignoring the ambiguous intervals of Doppler velocity between $V_{z m i n}$ and $-V_{0}(1+\lambda \cos 2 \varphi)>V_{z \min }$ and between $V_{0}(1+\lambda \cos 2 \varphi)<$ $V_{z \max }$ and $V_{z \max }$, we simply assign to each of the two associated $z$ values half the de-projected emissivity, thereby avoiding double-counting.

Figure 8 illustrates the dependence of $\chi_{a x i}^{2}$ on parameters $\lambda$ and $\varphi$ for the same simulated wind configurations as displayed in Figure 7 . The effective emissivity used to produce the simulated data-cube is of the form $1 / r^{2}$ for $0.5<r<5$ arcsec with no dependence on stellar latitude in order not to bias the evaluation of $\chi_{a x i}^{2}$ or, more precisely, not to complicate its interpretation. For $r>5$ arcsec, the effective emissivity is taken to cancel and for $r<0.5$ arcsec it is taken to be constant.

In all cases $\chi_{a x i}^{2}$ is minimal at the simulated value of $(\lambda, \varphi)$ but in some cases a broader region of the $(\lambda, \varphi)$ plane is observed to be equally acceptable. The minimum is better behaved along the descending diagonal (wind velocities out of the sky plane) than along the ascending diagonal (wind velocities near the sky plane): in the former case $\chi_{a x i}^{2}$ is more efficient than $Q$ to constrain the $(\lambda, \varphi)$ pair while in 
the latter case $Q$ is more efficient than $\chi_{a x i}^{2}$.

Using $\varphi=0$ for de-projection deserves a special comment; in this case, a given circle $\left(R^{\prime}, z^{\prime}\right)$ is made of points having all the same value of $z=z^{\prime}$ and the same value of $R=R^{\prime}$, therefore a same value of $V_{z}$ and a same value of $d V_{z} / d z: \chi_{a x i}^{2}$ is simply a measure of the axi-symmetry of the data-cube about the $z$ axis for Doppler velocities $\left|V_{z}\right|<(1+\lambda) V_{0}$. In particular, when the real wind and the wind used for de-projection are both axi-symmetric with respect to the $z$ axis, with respective prolateness $\lambda_{\text {true }}$ and $\lambda_{\text {deproj }}, \chi_{a x i}^{2}=0$ is of no help to constrain the value of $\lambda_{\text {true }}$. The same is true of the study of $V_{z \min }, V_{z \max }$ and $Q$, in the approximation where the wind velocity and mass loss rate do not depend on $r$ : whatever the value of $\lambda$ the extrema of the velocity spectrum are reached in all pixels at a same value of $V_{z \max }$ and $V_{z \min }=-V_{z \max }$. The result of the de-projection depends on the location of $\lambda_{\text {true }}$ and $\lambda_{\text {deproj }}$ with respect to the value -0.2 that separates ambiguous from unambiguous cases. When both $\lambda_{\text {true }}$ and $\lambda_{\text {deproj }}$ are larger than -0.2 , the maximum of $\left|V_{z}\right|$ is reached at the poles and the value of $V_{0}$ used for de-projection is the true value multiplied by $\left(1+\lambda_{\text {true }}\right) /\left(1+\lambda_{\text {deproj }}\right)$. However, when one of the $\lambda$ parameters takes values that vary between -0.2 and -1 , the maximum of $\left|V_{z}\right|$ is reached at values of $|\sin \zeta|$ that vary between 1 and $1 / \sqrt{3}$, the corresponding values of $\left|V_{z}\right|$ varying between $0.8 V_{0}$ and $4 V_{0} /[3 \sqrt{3}] \sim 0.77 V_{0}$ with a minimum at $\lambda=-1 / 2$ where $|\sin \zeta|=1 / \sqrt{2}$ and $\left|V_{z}\right|=1 / \sqrt{2} V_{0} \sim 0.71 V_{0}$. This near independence on $\lambda\left(\sim \pm 3 V_{0} / 4\right)$ of $V_{z \min }$ and $V_{z \max }$ when $\varphi=0$ and $\lambda<-0.2$ could already be seen in Figure 6 . In general, when using $\varphi=0$ and $\lambda<-0.2$ for de-projection of a $\left(\lambda_{0}, \varphi_{0}\right)$ data-cube located in the unambiguous region will require the use of a $V_{0}$ value significantly larger than the true value and will deproject the large values of $\left|V_{z}\right|$, associated with large values of $|\sin \zeta|$, to intermediate values of $|\sin \zeta|$ resulting in relatively low values of $\chi_{a x i}^{2}$.

\section{CASE STUDIES: RS Cnc AND EP Aqr}

In the preceding sections, arguments were developed using simulated rather than real observations. In practice, the morphology of circumstellar envelopes of evolved stars are far from being as smooth and well-behaved as those simulated in the present study. The question of the practicability of using the arguments and the tools developed in the preceding sections remains therefore open at this stage. While each particular case must be considered separately, we find it useful to devote the present section to two case studies in order to get some idea of the nature and magnitude of the difficulties that one may have to face.

\subsection{RS Cnc}

The first star selected for this purpose is RS Cnc, an S-type AGB star (CSS 589 in Stephenson's 1984 catalogue) in the thermally-pulsing phase (Lebzelter \& Hron 1999), which can be considered as representative of its family, the morphology of its envelope being quite clumpy but not excessively. Analyses of Plateau de Bure observations of its $\mathrm{CO}(1-0)$ and $\mathrm{CO}(2-1)$ emissions have been published earlier (Libert et al. 2010; Hoai et al. 2014; Nhung et al. 2015a; Le Bertre et al. 2016). Here, we use CO(2-1) observations from the upgraded Plateau de Bure interferometer (NOEMA) having a beam size (FWHM) of $0.44 \times 0.28$ $\operatorname{arcsec}^{2}$ and a spectral resolution of $\sim 0.2 \mathrm{~km} \mathrm{~s}^{-1}$. The noise level per data cube element is $1.6 \mathrm{mJy}^{\text {beam }}{ }^{-1}$ for pixels of $0.07 \times 0.07 \operatorname{arcsec}^{2}$. In order to improve the signal to noise ratio, we limit the study to the region $R<4$ arcsec and we group pixels by $3 \times 3=9$, meaning $0.21 \times 0.21 \operatorname{arcsec}^{2}$; we use as experimental uncertainty on the brightness the quadratic sum of the noise and of $20 \%$ of the measured value. Figure 9 displays sky maps of the measured intensity multiplied by $R$ and of the mean Doppler velocity, together with the integrated Doppler velocity spectrum.

As already apparent from the map of the mean Doppler velocity, the projection of the star axis on the sky plane is nearly north-south oriented. Indeed we find that $\chi_{\text {rot }}^{2}$ is minimal for $\theta_{0} \sim 7^{\circ}$ and we display in Figure 10 (left) the dependence on $\theta-\theta_{0}$ of its normalized value (divided by the number of degrees of freedom). The value of $\chi_{\text {rot }}^{2}$ at minimum, $\sim 4$, is the result of the relative lumpiness of the measured brightness. The uncertainty on $\theta$ is accordingly poorly defined; as an indication of how well $\theta$ is measured we quote as effective uncertainty the value associated with a $10 \%$ increase of $\chi_{\text {rot }}^{2}$ with respect to minimum, namely $\theta$ $=7^{\circ} \pm 10^{\circ}$. Indeed, masking measured brightness values below noise level (one sigma) minimizes $\chi_{\text {rot }}^{2}$ at $\theta \sim 10^{\circ}$ instead of $\sim 7^{\circ}$

Having obtained an evaluation of $\theta$ we rotate the datacube by $7^{\circ}$ about the line of sight in order to have effectively $\theta=0$ and proceed with the evaluation of the scale $V_{0}$ of the wind velocity and the exclusion from the $(\lambda, \varphi)$ plane of regions unsuited for de-projection as was done in Section 5 using simulated data. The dependence of $Q$ on $\lambda$ and $\varphi$ is displayed in the central panel of Figure 10. At first sight, the resemblance with the upper-middle panel of Figure 7 ( $\left.\lambda=0.4, \varphi=45^{\circ}\right)$ is striking; however, the minimum is now considerably less steep than it was for simulated data and extends over a broad region that excludes wind velocities closely confined to the sky plane, whether bipolar or equatorial outflows. The resulting $V_{0}$ values are displayed in the right panel of Figure 10 and range between $\sim 4$ and $\sim 15 \mathrm{~km} \mathrm{~s}^{-1}$, the lower values being associated with prolate bipolar outflows near the line of sight or oblate equatorial outflows having their axis near the sky plane, namely wind configurations in the unambiguous de-projection region. The larger values are instead confined to the ambiguous de-projection region of the $(\lambda, \varphi)$ plane.

A major cause of error in the evaluation of $V_{0}$ is the difficulty to measure accurately $V_{z \min }$ and/or $V_{z \max }$ in pixels where the brightness is close to noise level at the extremities of the Doppler velocity spectrum. In calculating in each pixel the values of $V_{z \min }$ and $V_{z \max }$ we used brightness in excess of two noise $\sigma$ 's and checked that the result was essentially unaffected when using instead one or three noise $\sigma$ 's. The left panels of Figure 11 display distributions of the values obtained for $V_{0}$ in each pixel (two values per pixel) for some representative values of the $(\lambda, \varphi)$ pair. Ideally, if the selected wind configuration describes the data well, the $V_{0}$ distribution must have the shape of a narrow peak centered at the proper $V_{0}$ value. Such a peak is indeed visible for some 

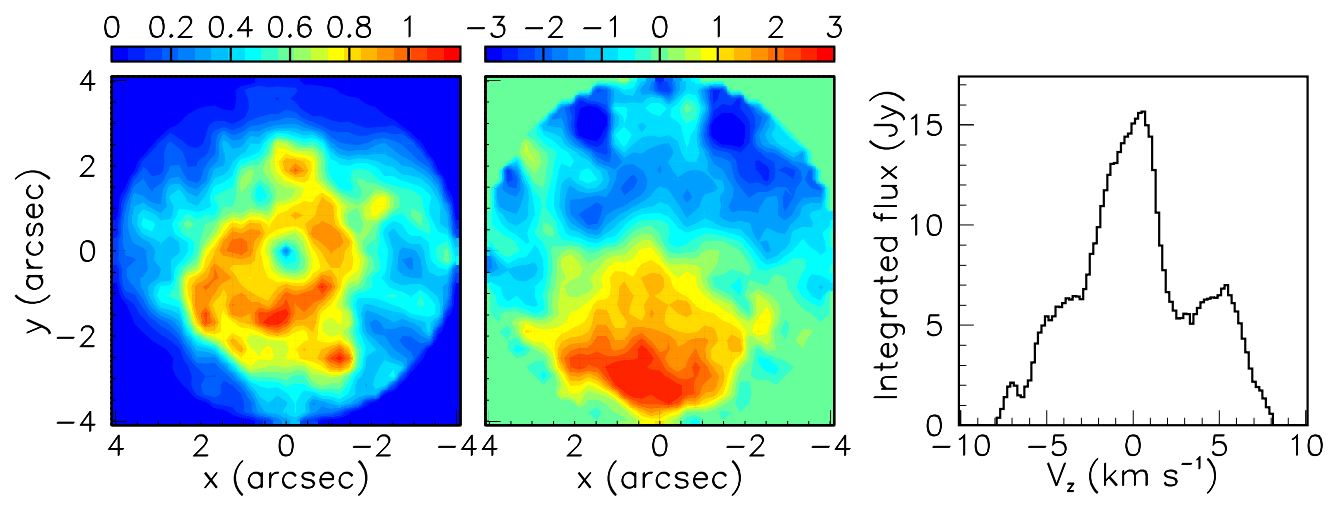

Figure 9. NOEMA observations of the $\mathrm{CO}(2-1)$ emission of RS Cnc in the region $R<4$ arcsec. Left: sky map of the intensity (Jy beam ${ }^{-1}$ ) multiplied by $R$ (arcsec). Middle: sky map of $\left\langle V_{z}\right\rangle\left(\mathrm{km} \mathrm{s}^{-1}\right)$. Right: Doppler velocity $V_{z}$ spectrum $\left(\mathrm{km} \mathrm{s}^{-1}\right)$ integrated over the region $R<4$ arcsec. In the first two panels north is up and east is left.
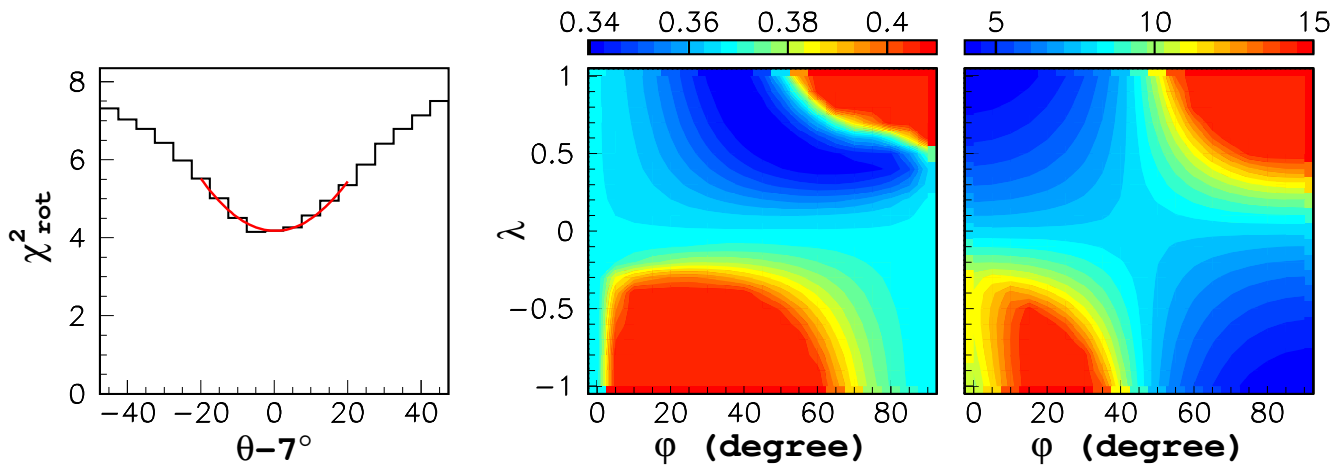

Figure 10. RS Cnc $\mathrm{CO}(2-1)$ emission. Left: dependence of $\chi_{\text {rot }}^{2}$ on $\theta-7^{\circ}$; the curve is a parabolic fit. Middle: $(\lambda, \varphi)$ map of $Q$ for the region $R<4$ arcsec. The colour scale runs from minimum $(\sim 0.34)$ to 1.2 times minimum. Right: $(\lambda, \varphi)$ map of the velocity scale $V_{0}(\mathrm{~km}$ $\left.\mathrm{s}^{-1}\right)$.

values of the $(\lambda, \varphi)$ pairs, but it is broad and accompanied by a low $V_{0}$ tail: exploiting the information contained in the dependence over the sky plane of $V_{z \min }$ and $V_{z \max }$ is clearly more difficult when dealing with real data than it is with simulated data. In pixels where the brightness is close to noise level at the extremities of the Doppler velocity spectrum $\left|V_{z \min }\right|$ and/or $\left|V_{z \max }\right|$ are under-evaluated and the same is therefore true for $V_{0}$. Pixels at larger values of $R$, being associated with lower intensities, are more likely to be of that kind. Indeed, as can be seen in Figure 11, the distribution of $V_{0}$ obtained inside the circle $R<3 \operatorname{arcsec}$ is narrower and less contaminated by a low $V_{0}$ tail than inside the circle $R<4$ arcsec. The distributions displayed in the left panels of Figure 11 suggest that a figure of merit revealing the presence of a peak in the $V_{0}$ distribution might be more efficient than $Q$ in exploiting the information carried by $V_{z \min }$ and $V_{z \max }$. We use a simple algorithm to evaluate, for each value of the $(\lambda, \varphi)$ pair the peak to tail ratio of the $V_{0}$ distribution, $P / T$. The distribution of $P / T$ over the $(\lambda, \varphi)$ plane is displayed in Figure 11 (right). It is consistent with the information carried by $Q$ but is much more selective: a reasonable cut $Q>0.36$ is only a factor 1.06 above minimum while an equivalent cut $P / T<2$ is a factor $\sim 4$ above minimum and a factor 2.5 below maximum, allowing for safely rejecting regions of the $(\lambda, \varphi)$ plane having $P / T<2$.

In order to further restrict the acceptable region of the $(\lambda, \varphi)$ plane, we still need to exploit the constraint of axisymmetry as was done in Section 6 using simulated observations. This, however, cannot be done reliably in the regions of ambiguous de-projection (right panel of Figure 3). As these have a large overlap with the regions disfavoured by the analysis of the $(\lambda, \varphi)$-dependence of $V_{z \min }$ and $V_{z \max }$, we may exclude them from the analysis by rejecting regions containing a "harmful" extremum of the $V_{z}$ vs $z$ relation as defined in the right panel of Figure 3, namely regions where $d V_{z} / d_{z}$ cancels in the $|\sin \zeta|<0.9$ interval. When an extremum occurs at $|\sin \zeta|>0.9$, we share the de-projected emissivity equally between the two associated values of $\sin \zeta$. The distribution of $\chi_{a x i}^{2}$ in the $(\lambda, \varphi)$ plane is displayed in Figure 12 together with the boundaries associated with regions containing a "harmful" extremum and with the con- 

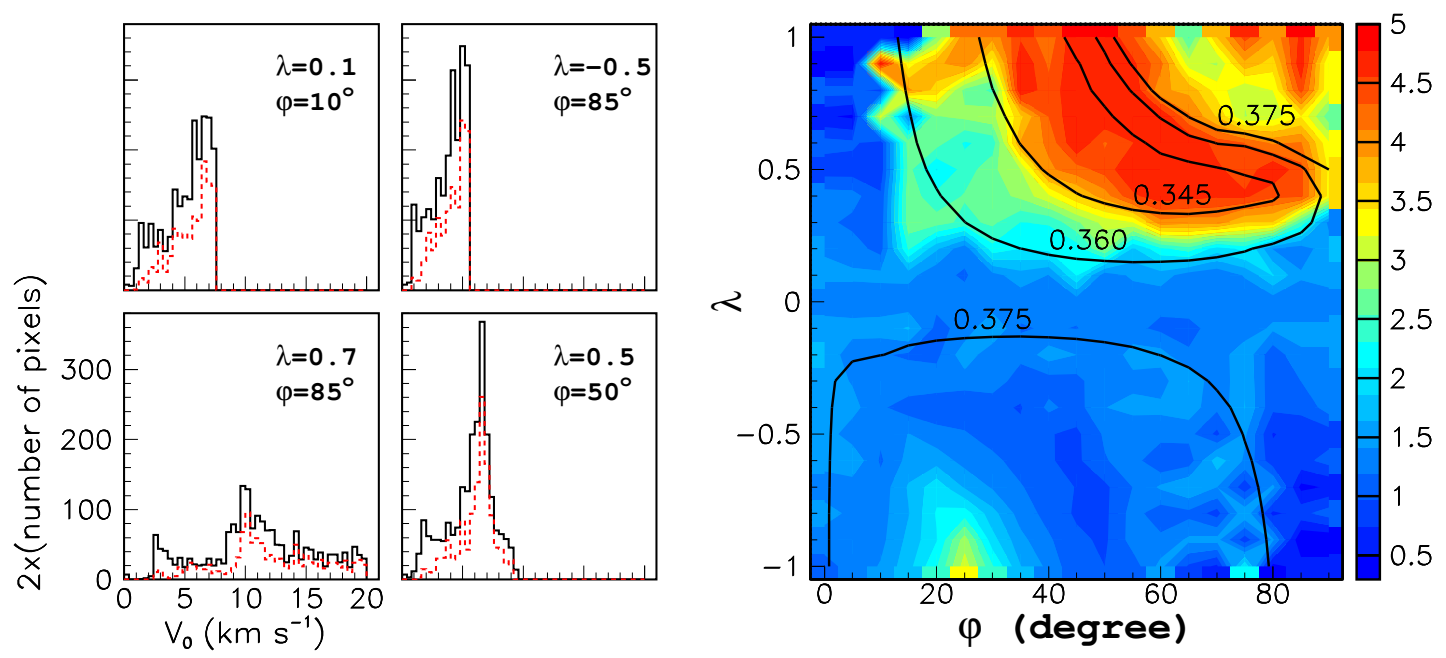

Figure 11. RS Cnc. Left: $V_{0}$ distributions obtained for four different values of the $(\lambda, \varphi)$ pair and for $R<4$ arcsec (solid) or $R<3$ $\operatorname{arcsec}($ dashed). Right: distribution of $P / T$ in the $(\lambda, \varphi)$ plane for $R<3 \operatorname{arcsec}$. The contours correspond to $Q=0.345,0.360$ and 0.375 respectively.

tours associated with $Q=0.36$ and $P / T=2$. The main contribution of $\chi_{a x i}^{2}$ is to disfavour wind configurations close to spherical with an axis at intermediate inclination with respect to the sky plane, leaving a relatively narrow region of acceptable bipolar outflows in the upper-left quadrant of the $(\lambda, \varphi)$ plane with $\lambda$ between $\sim 0.3$ and $\sim 0.8$ and $\varphi$ between $\sim 15^{\circ}$ and $\sim 45^{\circ}$.

The joint analysis of $\mathrm{CO}(1-0)$ and $\mathrm{CO}(2-1)$ emissions presented in Nhung et al. (2015a) gave $\left(V_{0}, \lambda, \theta, \varphi\right)=(5.0 \pm$ $\left.0.2 \mathrm{~km} \mathrm{~s}^{-1}, 0.50 \pm 0.02,9^{\circ} \pm 6^{\circ}, 38^{\circ} \pm 2^{\circ}\right)$, however allowing for some $\mathrm{r}$-dependence of the expansion velocity. This corresponds indeed to values of $Q<0.36, P / T>2$ and $\chi_{a x i}^{2}<10$ favoured by the present study. We display in Figure 12 (right) the dependence of the de-projected emissivity, multiplied by $r^{2}$ on respectively $r, \omega$ and $z^{\prime} / r=\sin \alpha$ for three different values of the $(\lambda, \varphi)$ pair in the favoured region, $(\lambda, \varphi)=\left(0.5,40^{\circ}\right),\left(0.6,30^{\circ}\right)$ and $\left(0.7,20^{\circ}\right)$ respectively. All three distributions are averaged in the sphere $r<4$ arcsec. A remarkable result is the independence of the $r$-dependence of the de-projected effective emissivity on the value of $\left(V_{0}, \lambda, \varphi\right)$ used for de-projection, a result that had been anticipated and explained in Section 2 using Relations 5 . However, the longitudinal dependence differs significantly from uniform, with an excess in the $180^{\circ}<\omega<360^{\circ}$ hemisphere compared with $0<\omega<180^{\circ}$. The latitudinal dependence displays a clear asymmetry with respect to the star equator. Table 1 summarizes the results.

Commenting further on these results goes beyond the scope of the present study. Physics arguments need now to be used for interpreting the observed behaviour of the deprojected emissivity and its relation with the probably much too simple form assumed for the wind configuration. Yet, the present results are of considerable help in constructing a physics model of the morphology and kinematics of the circumstellar envelope and have provided a deep insight in the constraints that such a model has to obey.

\subsection{EP Aqr}

The second star used for illustration is EP Aqr, an oxygenrich $\mathrm{M}$ type AGB star that is probably at the beginning of its evolution on the thermally pulsing phase (Lebzelter \& Hron 1999; Cami et al. 2000) in spite of observations of trailing gas (Cox et al. 2012; Le Bertre \& Gérard 2004) suggesting a mass loss episode at the scale of $10^{4}$ to $10^{5}$ years. Recently, observations of ${ }^{12} \mathrm{CO}(1-0)$ and ${ }^{12} \mathrm{CO}(2-1)$ emissions using the IRAM 30-m telescope and the Plateau de Bure Interferometer have been reported (Winters et al. 2003, 2007; Nhung et al. 2015b; Le Bertre et al. 2016). Here, we use $\mathrm{CO}(1-0)$ and $\mathrm{CO}(2-1)$ observations made in Cycle 4 of ALMA operation (Nhung et al. 2018, 2016.1.00026.S). The beam size $(\mathrm{FWHM})$ is respectively $0.78 \times 0.70$ and $0.33 \times 0.30$ $\operatorname{arcsec}^{2}$ and the noise respectively 8 and $7 \mathrm{mJy} \mathrm{beam}^{-1}$ for pixels of respectively $0.2 \times 0.2$ and $0.1 \times 0.1 \operatorname{arcsec}^{2}$ and Doppler velocity bins of $0.2 \mathrm{~km} \mathrm{~s}^{-1}$. Similar but different ALMA observations, including also $\mathrm{SiO}$ and $\mathrm{SO}_{2}$ emission, have recently been presented by Homan et al. (2018).

We restrict the analysis to the sky plane region having $R<8$ arcsec. Figure 13 displays sky maps of the deviation from unity of the ratio $\Delta(x, y)=F(x, y) /\langle F(x, y)\rangle$ between the measured intensity $F(x, y)$ and its average $<F(x, y)>$ over position angle. It gives evidence for approximate isotropy in the sky plane, suggesting that the wind is either spherical or axi-symmetric with the symmetry axis near the line of sight. Indeed, $\chi_{\text {rot }}^{2}$ is found to display a very broad minimum, centered at $\theta_{0} \sim-20^{\circ}$ for $\mathrm{CO}(1-0)$ emission and at $\theta_{0} \sim-31^{\circ}$ for $\mathrm{CO}(2-1)$ emission; its dependence on $\theta-\theta_{0}$ is shown in Figure 14. Using the same criterion as for RS Cnc to measure the uncertainty on its measurement, namely a $10 \%$ increase of $\chi_{\text {rot }}^{2}$, gives $\theta=-20^{\circ} \pm 37^{\circ}$ and $-31^{\circ} \pm 43^{\circ}$ respectively, consistent with a common value of $-25^{\circ} \pm 28^{\circ}$. The agreement between the $\mathrm{CO}(1-0)$ and $\mathrm{CO}(2-$ 1) results suggests that the deviation from sphericity is real 

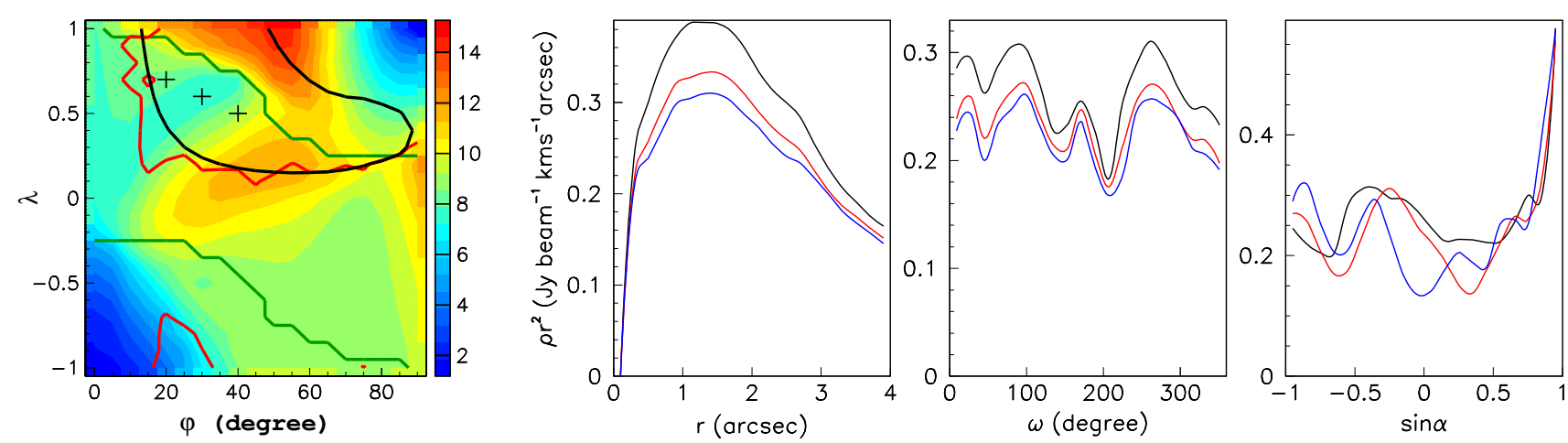

Figure 12. RS Cnc. Left: distribution of $\chi_{a x i}^{2}$ in the $(\lambda, \varphi)$ plane. The contours are for ambiguous de-projection (green), $Q=0.36$ (black) and $P / T=2$ (red). Rightmost panels (from left to right): dependence of the de-projected emissivity multiplied by $r^{2}$ (Jy beam ${ }^{-1} \mathrm{~km}$ $\mathrm{s}^{-1}$ arcsec) on $r$ (arcsec), $\omega$ (degree) and $\sin \alpha$ respectively. The values of $(\lambda, \varphi)$ are, from up down, $\left(0.5,40^{\circ}\right),\left(0.6,30^{\circ}\right)$ and $\left(0.7,20^{\circ}\right)$, corresponding to the crosses in the left panel.

Table 1. De-projection of RS Cnc CO(2-1) observations: summary of results

\begin{tabular}{|c|c|c|c|c|c|c|c|}
\hline Case & $V_{0}(\mathrm{~km} / \mathrm{s})$ & $\lambda$ & $\varphi\left(^{\circ}\right)$ & $\theta\left(^{\circ}\right)$ & $Q$ & $P / T$ & $\chi_{a x i}^{2}$ \\
\hline Nhung et al. (2015a) & $5.0 \pm 0.2$ & $0.50 \pm 0.02$ & $38 \pm 2$ & $9 \pm 6$ & - & - & - \\
\hline 1 & 6.0 & 0.5 & 40 & 7 & 0.344 & 3.6 & 9.5 \\
\hline 2 & 5.0 & 0.6 & 30 & 7 & 0.345 & 2.0 & 7.5 \\
\hline 3 & 4.5 & 0.7 & 20 & 7 & 0.356 & 2.2 & 8.7 \\
\hline
\end{tabular}

and the broad distribution of $\chi_{\text {rot }}^{2}$ as a function of $\theta-\theta_{0}$ indicates that the star axis must make a small angle with the line of sight. Also shown in Figure 14 (middle panels) is the dependence of $Q$ on $\lambda$ and $\varphi$. It remains nearly constant over a very large region of the $(\lambda, \varphi)$ plane. The reason is that $V_{z \min }$ and $V_{z \max }$ are nearly invariant over the sky plane. In order to quantify better this statement, we integrate the Doppler velocity spectrum between $R=1$ arcsec and $R=8$ arcsec and display it in 18 bins of position angle $\psi$, each $20^{\circ}$ wide. The high sensitivity that results allows for measuring in each bin $V_{z \min }$ and $V_{z \max }$ with a precision of $\sim 0.1 \mathrm{~km} \mathrm{~s}^{-1}$. We obtain this way measurements of the width $W_{v z}$ and the offset $\Delta_{v z}$ of the Doppler velocity spectrum in each bin of $\psi$. Their mean and rms values for $\mathrm{CO}(1-0)$ and $\mathrm{CO}(2-1)$ emission respectively, relative to the spectrum width, are $\left\langle W_{v z}\right\rangle=21.30 \mathrm{~km} \mathrm{~s}^{-1}$ and 21.61 $\left.\mathrm{km} \mathrm{s}^{-1}, \operatorname{Rms}\left(W_{v z}\right) /<W_{v z}\right\rangle=2.110^{-2}$ and $2.910^{-2}$ and $\operatorname{Rms}\left(\Delta_{v z}\right) /\left\langle W_{v z}\right\rangle=1.210^{-2}$ and $2.010^{-2}$ respectively. The latter two quantities are compared with the predictions of the simple model in the right panels of Figure 14. Compared with $Q$ they are more reliable discriminants in rejecting regions of the $(\lambda, \varphi)$ plane but leave a very broad acceptable region along the descending diagonal. The situation here is very different from what was found for RS Cnc: it would not have helped to use a discriminant such as $P / T$ because the $V_{0}$ distribution is extremely narrow in the low $Q$ region of the $(\lambda, \varphi)$ plane. Figure 15 displays the dependence of $\chi_{a x i}^{2}$ on $\lambda$ and $\varphi$. We use as uncertainty on the measured brightness the quadratic sum of $20 \%$ of its value and of the noise. For both $\mathrm{CO}(1-0)$ and $\mathrm{CO}(2-1)$ emissions, large values of $\varphi$ are excluded; taking into account the constraint imposed by the study of $V_{z \min }$ and $V_{z \max }$ leaves a large region in the upper left quadrant of the map acceptable for de-projection. The result obtained earlier by Nhung et al. (2015b), $\left(V_{0}, \lambda, \theta, \varphi\right)=\left(6.0 \mathrm{~km} \mathrm{~s}^{-1}, 0.67,-36^{\circ}\right.$, $\left.13^{\circ}\right)$ is contained in this region. We select three representative $(\lambda, \varphi)$ pairs for purpose of illustration: $\left(0,0^{\circ}\right),\left(0.35,6^{\circ}\right)$ and $\left(0.7,12^{\circ}\right)$. The corresponding parameters are listed in Table 2 and the dependence on $r, \omega$ and $\sin \alpha$ of the associated de-projected effective emissivity multiplied by $r^{2}$ inside the sphere $r<8$ arcsec is displayed in Figure 16. The difference between the $\mathrm{CO}(1-0)$ and $\mathrm{CO}(2-1)$ radial distributions is understood as being caused by the different temperature dependence (Nhung et al. 2018). The smaller $\lambda$, the larger $V_{0}$ and the stronger the concentration of the effective emissivity near the equatorial plane. Indeed one evolves from a bipolar outflow for $\lambda=0.7$ with no significant equatorial enhancement of effective emissivity to a spherical wind producing a strong enhancement of effective emissivity at equator for $\lambda=0$. Only physics arguments can help choosing between the possible wind configurations. In particular, the presence of a companion star or massive planet in the equatorial plane would probably favour an enhancement of the effective emissivity while not making the wind velocity much deviate from spherical. A bipolar wind associated with an isotropic effective emissivity would be more difficult to explain. However, such arguments are well beyond the scope of the present article.

\section{SUMMARY AND CONCLUSION}

The above analysis of the problem of de-projection of radially expanding axi-symmetric circumstellar envelopes has provided a deep insight into its main features and has devised tools that help with its solution. However, 

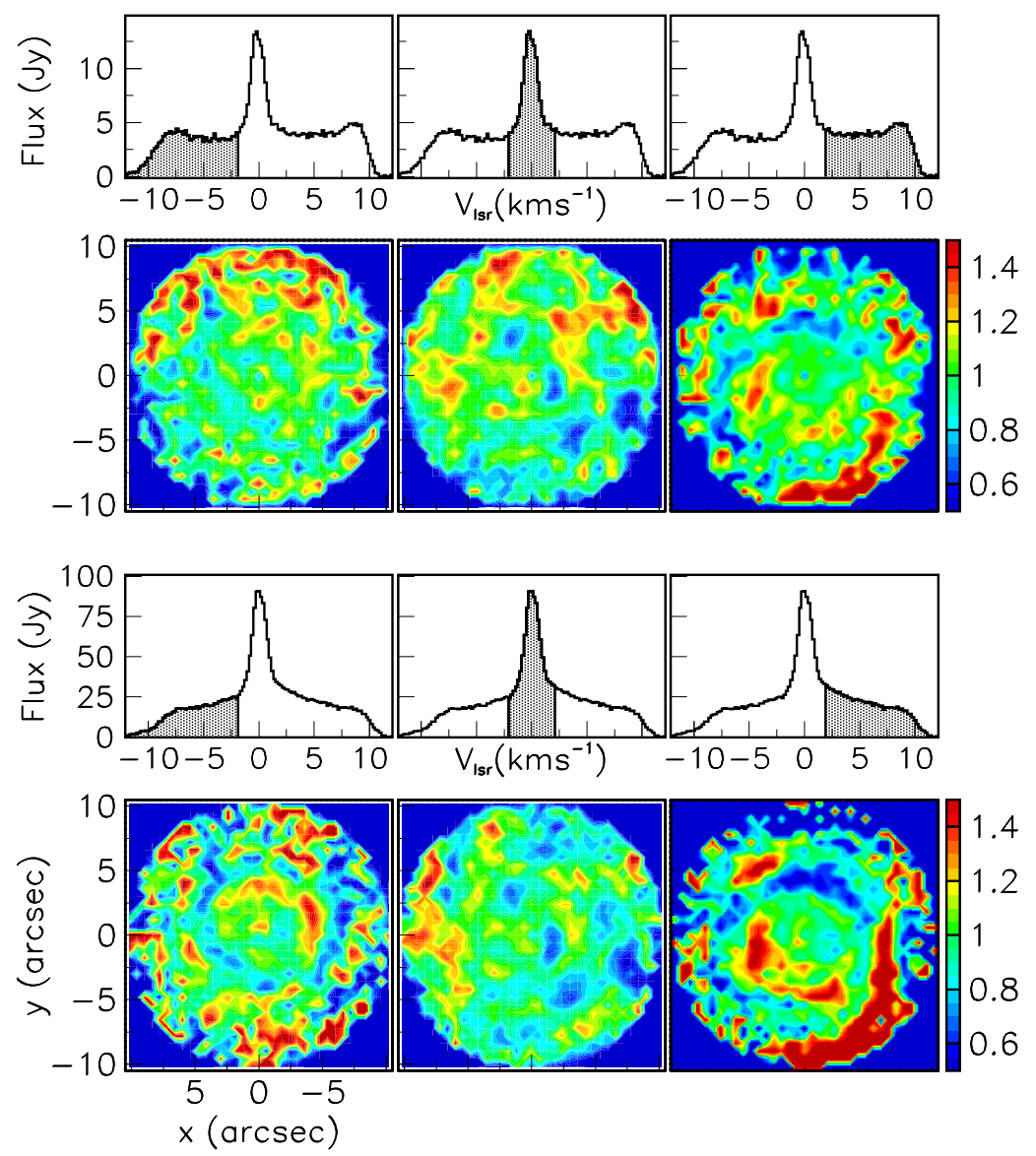

Figure 13. EP Aqr CO(1-0) (upper panels) and $\mathrm{CO}(2-1)$ (lower panels) emission. Sky maps of $\Delta(x, y)-1$ for $R<10$ arcsec. Left panels are for $V_{z}<-2 \mathrm{~km} \mathrm{~s}^{-1}$, central panels for $\left|V_{z}\right|<2 \mathrm{~km} \mathrm{~s}^{-1}$ and right panels for $V_{z}>2 \mathrm{~km} \mathrm{~s}^{-1}$ as indicated in the Doppler velocity spectra displayed above the sky maps. From Nhung et al. (2018).

Table 2. De-projection of EP Aqr CO(1-0) observations: summary of results

\begin{tabular}{|c|c|c|c|c|c|c|c|}
\hline Emission & Case & $V_{0}\left(\mathrm{~km} \mathrm{~s}^{-1}\right)$ & $\lambda$ & $\varphi\left(^{\circ}\right)$ & $\theta\left(^{\circ}\right)$ & $Q$ & $\chi_{a x i}^{2}$ \\
\hline $\mathrm{CO}(1-0) \&(2-1)$ & Nhung et al. $(2015 \mathrm{~b})$ & 6.0 & 0.67 & 13 & -36 & - & - \\
\hline \multirow{3}{*}{$\mathrm{CO}(1-0)$} & 1 & 10.6 & 0 & 0 & \multirow{3}{*}{0} & \multirow{3}{*}{0.29} & 0.44 \\
\cline { 2 - 6 } & 2 & 8.0 & 0.35 & 6 & 0.57 \\
\cline { 2 - 5 } & 3 & 6.5 & 0.7 & 12 & & & 0.66 \\
\hline \multirow{3}{*}{$\mathrm{CO}(2-1)$} & 1 & 10.8 & 0 & 0 & \multirow{3}{*}{-31} & \multirow{3}{*}{0.37} & 0.68 \\
\cline { 2 - 5 } & 2 & 8.1 & 0.35 & 6 & & 0.89 \\
\cline { 2 - 5 } & 3 & 6.6 & 0.7 & 12 & & & 1.00 \\
\hline
\end{tabular}

it does not offer a substitute to a detailed analysis that takes into account the physics of the mechanisms at play, such as hydrodynamical constraints and radiative transfer considerations. It is only meant to shed light on some of its intricacies and to set the frame for preliminary considerations that can help the construction of a realistic physical model. The effective emissivity used throughout the paper to describe observations is a convenient quantity that offers simplicity but hides the difficulty of disentangling the effects of temperature from those of density. Its assumed axi-symmetry is often a good approximation but is only valid for envelopes that are sufficiently optically thin and the assumption of radial expansion, implying the absence of rotation, is often violated in the later stages of the star evolution on their way to planetary nebulae. It is therefore essential to keep in mind the limited scope of the results of the present work.

With these caveats in mind, we summarize below the main results that have been obtained:

i) The position angle $\theta$ of the projection of the star axis on the sky plane has been shown to minimize a quantity, $\chi_{\text {rot }}^{2}$, independently from the particular form taken by the effective emissivity; its minimization, as long as it is made over the whole data-cube and not simply on its projection on the sky plane, makes optimal use of the available information. Only in cases where the problem has no solution, either because the star axis is close to the line of sight or because the wind velocity and the effective 

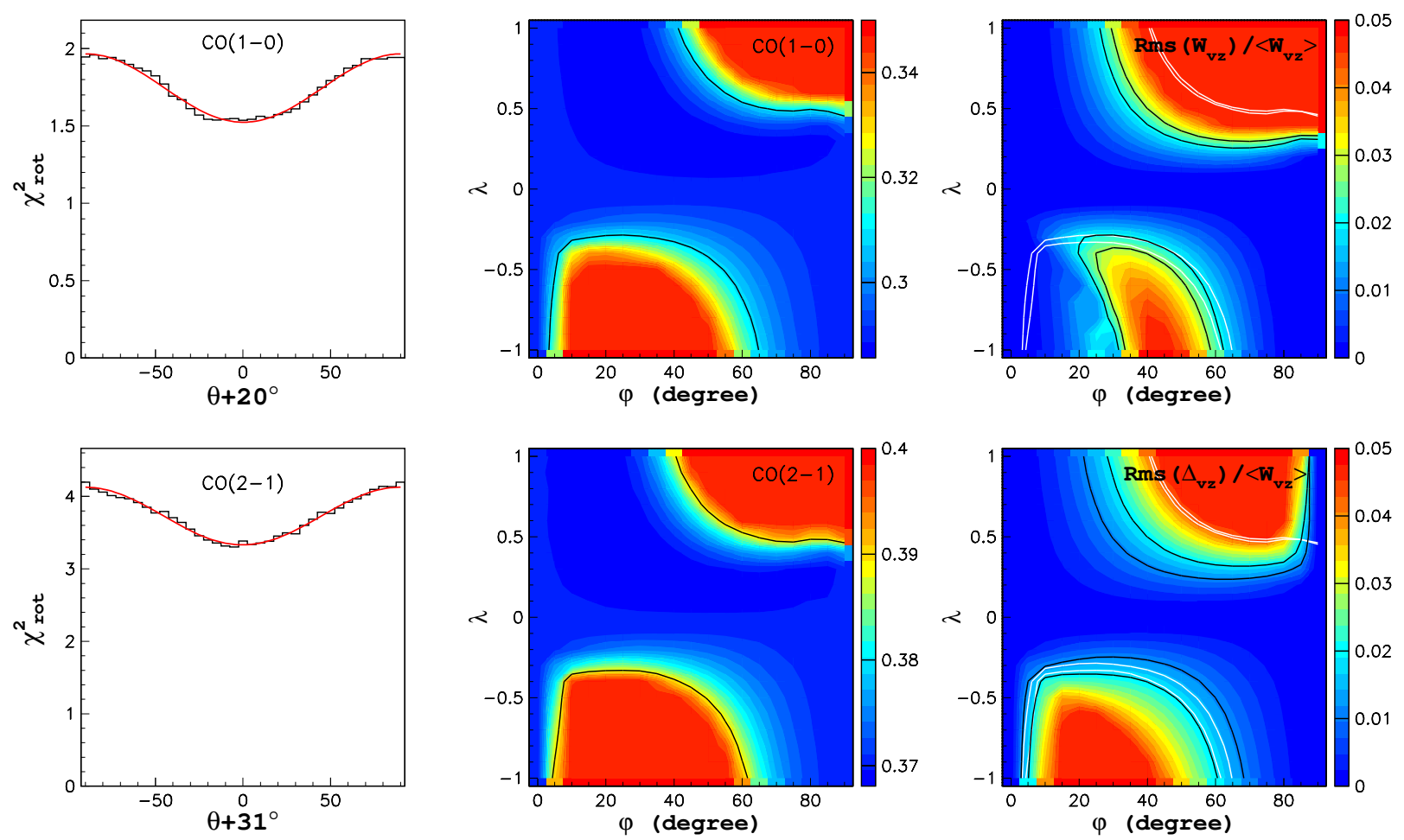

Figure 14. EP Aqr $\mathrm{CO}(1-0)$ and $\mathrm{CO}(2-1)$ emission. Left: dependence of $\chi_{\text {rot }}^{2}$ on $\theta-\theta_{0}$; the curves are cosine square fits. Middle: $(\lambda, \varphi)$ maps of $Q$ for the region $R<8$ arcsec. The contours show $Q=0.31$ for $\operatorname{CO}(1-0)$ and $Q=0.39$ for $\operatorname{CO}(2-1)$. Right: $(\lambda, \varphi)$ maps of $\operatorname{Rms}\left(W_{v z}\right) /<W_{v z}>$ and $\operatorname{Rms}\left(\Delta_{v z}\right) /<W_{v z}>$ as predicted by the simple model. The black contours display the values measured for $\mathrm{CO}(1-0)$ and $\mathrm{CO}(2-1)$ emission. The white contours are the same as shown in black in the middle panels.
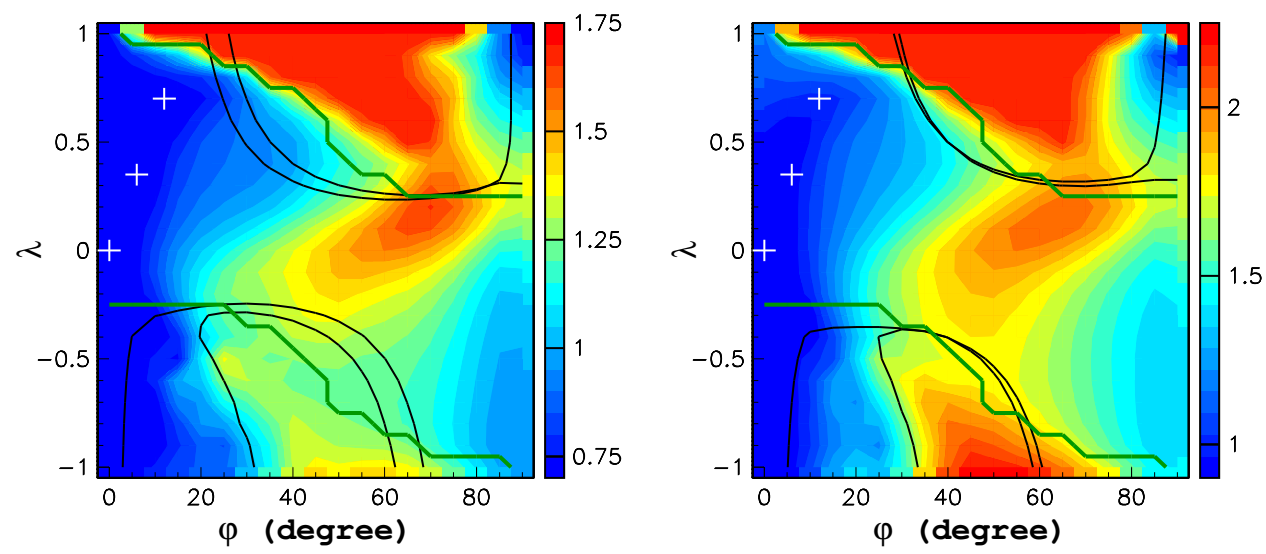

Figure 15. EP Aqr $\mathrm{CO}(1-0)$ (left) and $\mathrm{CO}(2-1)$ (right) emission. Dependence of $\chi_{a x i}^{2}$ on $\lambda$ and $\varphi$. On both panels the colour scale runs from minimum to 2.5 times minimum. The black contours indicate the regions rejected by $\operatorname{Rms}\left(W_{v z}\right) /\left\langle W_{v z}>\operatorname{and} \operatorname{Rms}\left(\Delta_{v z}\right) /<W_{v z}\right\rangle$. The green contours show the limits of ambiguous de-projection. Crosses indicate values of the $(\lambda, \varphi)$ pair listed in Table 2.

emissivity are nearly isotropic, is $\theta$ ill-defined.

ii) A good evaluation of the scale of the wind velocity $-V_{0}$ in the simple model-has been obtained from a study, in each pixel, of the dependence on position angle $\psi$ of the end points $V_{z \min }$ and $V_{z \max }$ of the Doppler velocity spectra. To this effect, the rms deviation of the velocity scale relative to its mean over the sky plane, $Q$, was found to be sufficient, in the case of simulated data, to quantify the agreement with a model. Its minimization helps in eliminating regions of the prolateness $(\lambda)$ versus inclination $(\varphi)$ plane which are unsuitable for de-projection. The method is particularly efficient when the wind velocity is confined near the sky plane, either as strongly prolate with axis close the sky plane or as 

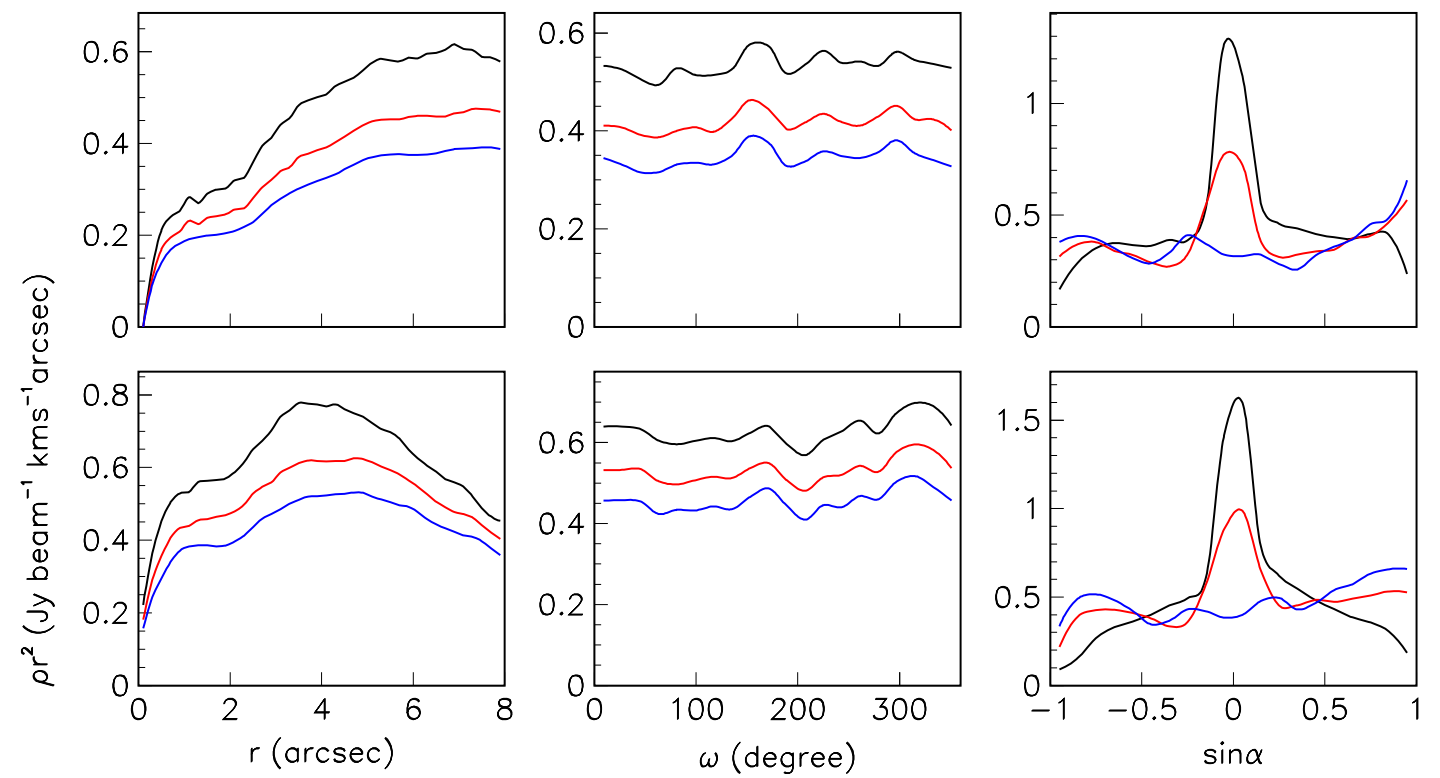

Figure 16. Dependence of the de-projected emissivity for EP Aqr multiplied by $r^{2}$ (Jy beam ${ }^{-1} \mathrm{~km} \mathrm{~s}^{-1} \operatorname{arcsec}$ ) on $r$ (arcsec, left), $\omega$ (degrees, middle) and $\sin \alpha$ (right) respectively. The values of $(\lambda, \varphi)$ are, from up down, $\left(0,0^{\circ}\right),\left(0.35,6^{\circ}\right)$ and $\left(0.7,12^{\circ}\right)$, corresponding to the crosses in Figure 15. Upper panels are for $\mathrm{CO}(1-0)$ emission and lower panels for $\mathrm{CO}(2-1)$ emission.

strongly oblate with axis close to the line of sight. However, in the case of real data, a more careful analysis is needed in order to exploit the richness of the information contained in the dependence of $V_{z \min }$ and $V_{z \max }$ on $\lambda$ and $\varphi$. A careful evaluation of $V_{z \min }$ and $V_{z \max }$, taking the noise level in proper account, is mandatory and algorithms allowing for separating the $V_{0}$ peak from a background of improper values may be helpful.

iii) Having obtained sensible estimates of $\theta$ and $V_{0}$, one can further constrain the $(\lambda, \varphi)$ pair by imposing axisymmetry on the de-projected effective emissivity. To this effect, a quantity $\chi_{a x i}^{2}$ has been constructed, which is minimal for maximal axi-symmetry. Contrary to the minimization of $Q$, the minimization of $\chi_{a x i}^{2}$ is inefficient when the wind velocity is confined near the sky plane. In such cases, the evaluation of the optimal value of the $(\lambda, \varphi)$ pair relies more on the constraints imposed by the distribution on the sky plane of the width and offset of the Doppler velocity spectra than on the constraints imposed by the requirement of axi-symmetry.

iv) Having obtained estimates of the orientation of the star axis, $\theta$ and $\varphi$, of the scale of the wind velocity, $V_{0}$, and of the effective prolateness of its distribution, $\lambda$, we are then in a position to de-project the effective emissivity as long as a single value of $z / r$ is associated with each bin of measured Doppler velocity. However, the effective emissivity cannot be directly de-projected in regions of $z / r$ that are associated with ambiguous velocity bins. Such regions are particularly important when the wind velocity is confined in the vicinity of the sky plane, implying that the Doppler velocity distribution is folded on itself and confined to lower values. In such cases, the observations measure mostly the projec- tion of the effective emissivity on the sky plane, namely the integrated brightness (or intensity).

v) To a good approximation, the $r$-dependence of the de-projected effective emissivity is obtained independently from the choice of the wind configuration used for deprojection as long as the wind velocity does not depend on $r$.

vi) As an illustration, we have presented two case studies of $\mathrm{CO}$ emission of AGB stars. They were chosen to be representative of typical observations rather than of the best space and spectral resolutions and sensitivity available today. In both cases, the method and tools developed in the present article have been shown to select efficiently wind configurations suitable for de-projection. Published analyses of the relevant observations have proposed models that are indeed favoured by these results. However, they also suggest exploring other wind configurations, of different inclination and prolateness, which may deserve being considered in the framework of a physics analysis. More importantly, they provide a deep understanding of the constraints imposed on a physics model and of how unique is the region of the $(\lambda, \varphi)$ plane ultimately selected.

vii) The main contribution of the present work may be the insight it has provided on the issue of the underdetermination of the problem of de-projection. It has underlined the importance of being conscious that a broad family of wind configurations, illustrated in blue in the right panel of Figure 3, can be used in principle to de-project the observed brightness data-cube into an effective emissivity at each point in space. In practice, however, constraints imposed by the requirement of axi-symmetry of the deprojected effective emissivity and of the need to populate in 
each pixel the totality of the observed Doppler velocity spectrum (but no more) have been found to complement each other; taken together, they are efficient in restricting the domain of the $(\lambda, \varphi)$ plane acceptable for de-projection. A good approximation to the $r$-distribution of the de-projected emissivity has been obtained under the hypothesis of constant velocity but physics considerations must then be used to decide how best to combine a possible velocity gradient with such $r$-dependence. More generally, the constraints imposed by hydrodynamics on the relation between density, temperature and velocity will be determinant in deciding on the "best" physics model.

\section{ACKNOWLEDGEMENTS}

We thank Professor Pierre Lesaffre for a careful reading of the manuscript and very useful and pertinent comments that helped improving significantly its content. This paper makes use of the following ALMA data: 2016.1.00026.S. ALMA is a partnership of ESO (representing its member states), NSF (USA) and NINS (Japan), together with NRC (Canada), NSC and ASIAA (Taiwan), and KASI (Republic of Korea), in cooperation with the Republic of Chile. The Joint ALMA Observatory is operated by ESO, AUI/NRAO and NAOJ. It also makes use of observations carried out with the IRAM NOEMA Interferometer and the IRAM 30 $m$ telescope. IRAM is supported by INSU/CNRS (France), MPG (Germany) and IGN (Spain). This research is funded by Graduate University of Science and Technology under grant number GUST.STS.DT2017-VL01. Financial and/or material support from the Vietnam National Space Center, the National Foundation for Science and Technology Development (NAFOSTED), the World Laboratory and Odon Vallet fellowships is gratefully acknowledged.

\section{REFERENCES}

Cami, J., Yamamura, I., de Jong, T., et al., 2000, A\&A, 360, 562 Cox, N. L. J., Kerschbaum, F., van Marle, A.-J., et al., 2012, A\&A, 537, A35

Diep, P. N., Phuong, N. T., Hoai, D. T. et al., 2016, MNRAS, 461,4276

Hoai D.T, Matthews, L.D., Winters, J.M. et al., 2014, A\&A, 565, A54

Höfner,S. \& Olofsson, H., Astron Astrophys Rev, 2018, 26, 1

Homan, W., Richards, A., Decin, L. et al., 2018, arXiv:1804.05684v2, accepted for publication in A\&A

Le Bertre, T., \& Gérard, E., 2004, A\&A, 419, 549

Le Bertre, T., Hoai, D.T., Nhung, P.T. and Winters, J.M., 2016, Conférence de la Société Française d'Astronomie et d'Astrophysique, L. Cambrésy et al. Eds.

Lebzelter, T. \& Hron, J., 1999, A\&A, 351, 553

Libert, Y., Winters, J.M., Le Bertre, T. et al. 2010, A\&A, 515, A112

Nhung, P.T., Hoai, D.T, Winters J.M., et al., 2015a, RAA, 15, 713

Nhung, P.T., Hoai, D.T., Winters, J.M. et al., 2015b, A\&A, 583, A64

Nhung, P.T., Hoai, D.T., Tuan-Anh, P. et al., 2018, submitted for publication in RAA

Stephenson, C. B., 1984, Publ. Warner \& Swasey Obs., 3, 1
Winters, J.M., Le Bertre, T., Jeong, K.S. et al., 2003, A\&A, 409, 715

Winters, J.M., Le Bertre, T., Pety, J.\& Neri, R., 2007, A\&A, 475, 559

This paper has been typeset from a $\mathrm{T}_{\mathrm{E}} \mathrm{X} / \mathrm{LAT}_{\mathrm{E}} \mathrm{X}$ file prepared by the author. 